\title{
Muster ethnischer Segregation in Deutschland - Ein Vergleich anhand räumlicher Segregationsmaße
}

Tobias Rüttenauer

Nuffield College

University of Oxford

New Road, Oxford OX1 1NF

United Kingdom

tobias.ruttenauer@nuffield.ox.ac.uk

\begin{abstract}
Zusammenfassung
Ethnische räumliche Segregation in Großstädten stellt eine mögliche Dimension der sozialen Abgrenzung zwischen Bevölkerungsgruppen dar und kann weitere Dimensionen der Ungleichheit in anderen Bereichen der Gesellschaft beeinflussen. Aufgrund unterschiedlicher räumlicher Einteilungen ist es oft jedoch schwierig das Ausmaß an Segregation über Stadtgrenzen hinweg zu vergleichen. Basierend auf Daten des Zensus 2011 verwendet dieser Beitrag räumliche Segregationsmaße auf verschiedenen geographischen Ebenen um so einen vergleichenden Überblick über die räumliche Segregation und deren Formen in Deutschland zu geben. Die Ergebnisse zeigen erhebliche Variation in der Stärke der räumlichen Trennung von Minderheiten. Außerdem verdeutlicht der Beitrag, dass verschiedene deutsche Städte unterschiedliche Muster der Segregation aufweisen. Während manche Städte besonders auf der kleinräumigen geographischen Ebene segregiert sind, erfolgt die Trennung in anderen Städten auf einer großräumigen Ebene. Dadurch zeigt der Beitrag, dass räumliche Segregation auch in Deutschland unterschiedliche Muster und Dimensionen annimmt, die mit unterschiedlichen Folgen und Ursachen verbunden sein können.
\end{abstract}

\section{KEYWORDS}

Segregation Germany, Spatial Segregation Indices, Comparison across cities, Ethnic Minorities

\section{Authors' pre-copy-editing version. Please cite the final chapter:}

Rüttenauer, T. (2021). Muster ethnischer Segregation in Deutschland - Ein Vergleich anhand räumlicher Segregationsmaße. In H. Kruse, J. Teltemann (Eds.) Differenz im Raum. Sozialstruktur und Grenzziehungen in deutschen Städten. Wiesbaden: Springer VS. Forthcoming 


\section{Einleitung}

Ein fundamentaler Bestandteil der sozialwissenschaftlichen Forschung ist die Untersuchung von (sozialen) Ungleichheiten. Diese Ungleichheiten umfassen z.B. die ungleiche Verteilung von Ressourcen, generelle Unterschiede im Lebenslauf, die ungleiche Beteiligung an Institutionen und gesellschaftlichen Entwicklungen, aber auch Ungleichheiten in räumlichen Prozessen. Räumliche Segregation beschreibt die ungleiche räumliche Verteilung von Bevölkerungsgruppen auf verschiedene geographische Gebiete innerhalb von Städten. Segregation bedeutet also, dass bestimmte Gruppen - ethnische Minderheiten, sozio-ökonomisch benachteiligte Haushalte oder demographische Gruppen - in einigen geographischen Bereichen der Stadt überproportional und in anderen unterproportional vertreten sind (vgl. auch Jähnen \& Helbig in diesem Band).

In den USA nimmt das Thema der räumlichen Segregation seit Jahrzehnten eine wichtige Rolle in der Sozialforschung ein (z.B. Firebaugh \& Farrell, 2016; Krysan \& Crowder, 2017; Massey \& Denton, 1993). Auch in Europa und Deutschland hat das Thema in den letzten Jahren an Bedeutung gewonnen (z.B. Friedrichs, 2008; Goebel \& Hoppe, 2015; Helbig \& Jähnen, 2018; Musterd, 2012; Teltemann, Dabrowski \& Windzio, 2015). Räumliche Segregation stellt deshalb eine wichtige Dimension der Ungleichheit dar, weil das räumliche Umfeld, in dem Personen aufwachsen und leben, potentiell einen großen Einfluss auf andere Bereiche des Lebens und damit auch auf andere Dimensionen der Ungleichheit hat: „Everything happens somewhere, which means that all action is embedded in place and may be a affected by its placement" (Logan, 2012, 508). So haben Studien zum Beispiel gezeigt, dass die räumliche Segregation Auswirkungen auf die Bildungschancen und die langfristige berufliche Entwicklung von Kindern (z.B. Chetty, Hendren \& Katz, 2016; Reardon, Kalogrides \& Shores, 2019; Sharkey \& Faber, 2014), Konflikte und Kriminalität in der Nachbarschaft (z.B. Legewie \& Schaeffer, 2016; Sampson, 2013, ; siehe auch Schaeffer, Jünger \& Klinger in diesem Band), die Belastung durch industrielle Umweltverschmutzung (z.B. Downey, 2007; Rüttenauer, 2019a), oder auch das allgemeine Wohlbefinden hat (z.B. Firebaugh \& Schroeder, 2009; Ludwig et al., 2012). Auch die Assimilation und Integration von Minderheiten und zugewanderten Gruppen in die Aufnahmegesellschaft hängt stark mit der räumlichen Trennung innerhalb der Städte zusammen (z.B. Alba \& Nee, 2005; Damm, 2009; Logan \& Zhang, 2010, ; siehe auch Dochow; Trittler \& Wöhler in diesem Band).

Schon diese selektiven Beispiele zeigen, dass räumliche Segregation einen Einfluss auf viele wichtige Dimensionen der Gesellschaft haben kann. Daher ist es wichtig, einen empirischen Überblick über das Ausmaß und die Dimensionen von räumlicher Segregation zu haben. Obwohl es für Deutschland mittlerweile eine Reihe von empirischen Studien gibt, die das Ausmaß an Segregation untersuchen (z.B. Friedrichs, 2008; Goebel \& Hoppe, 2015; Helbig \& Jähnen, 2018), ist es aus drei Gründen schwierig einen Vergleich zwischen deutschen Großstädten anzustellen: 1) Städte stellen die geographischen Merkmale der Bewohner auf Basis arbiträrer räumlicher Einheiten zur Verfügung (wie z.B. Stadtteile oder Bezirke) und herkömmliche Segregationsmaße reagieren sensitiv auf diese räumliche Einteilung. 2) Herkömmliche Segregationsmaße ignorieren die geographische Lage der Untersuchungseinheiten zueinander indem sie nur die reinen Anteile pro Einheit, nicht aber die geographische Lage dieser Einheiten zueinander berücksichtigen. 3) Segregation selbst kann theoretisch auf unterschiedlichen räumlichen Ebenen (Makro- und Mikro-Segregation) stattfinden. Dadurch sind herkömmliche Maße ungeeignet um unterschiedliche räumliche Muster der Segregation zu identifizieren.

Ziel dieses Beitrags ist es daher, anhand der einheitlich gegliederten Zensus Daten 2011 einen Vergleich des Ausmaßes der ethnischen räumlichen Segregation in Deutsch- 
land zu liefern. Des weiteren verwendet dieser Beitrag Segregationsmaße, welche die räumliche Struktur der demographischen Verteilung mit einbeziehen, um so nicht nur das Ausmaß an sich, sondern auch die räumlichen Muster der Segregation über die Städte hinweg zu vergleichen. Die Ergebnisse zeigen dabei nicht nur verschiedene Stärken an Segregation, sondern auch sehr unterschiedliche räumliche Muster. Während manche Städte besonders großräumig aufgeteilt sind und Minderheiten dabei in einigen Stadtteilen konzentriert wohnen, sind andere Städte besonders auf der kleinräumigen Ebene wie Straßenabschnitte oder Blöcke separiert. Theoretisch können diese unterschiedlichen Strukturen der Segregation auch qualitativ unterschiedliche Folgen für die Bewohner haben. So scheint es plausibel, dass kleinräumige Segregation einen stärkeren Einfluss auf alltägliche Interaktionen und wahrgenommene Diversität hat, während großräumige Muster eher den Zugang zu Ressourcen und Infrastrukturen oder auch die Stigmatisierung von Stadtteilen beeinflussen.

\section{Theorie}

Das Konzept der räumlichen Segregation hat in den Sozialwissenschaften eine lange Tradition und beschreibt allgemein in welchem Ausmaß zwei oder mehr Gruppen räumlich getrennt voneinander leben (Massey \& Denton, 1988). Obwohl diese Definition auf den ersten Blick relativ klar wirkt, gibt es eine Reihe von unterschiedlichen Konzepten, die dieser Definition gerecht werden. So kann dieses Ausmaß an Separierung durch eine Abweichung von der Gleichverteilung der Gruppen über eine Stadt hinweg beschrieben werden („evenness“). Aber auch Konzepte wie die Wahrscheinlichkeit des Kontakts zwischen Gruppen („exposure“), die räumliche Konzentration und die Cluster-Bildung von Gruppen („,concentration“ und „clustering“), oder die Zentralität innerhalb einer Stadt („centralization“) beschreiben weitere Dimensionen der räumlichen Separierung oder Segregation (Massey \& Denton, 1988). Welche dieser Dimensionen das Konzept der Segregation besser beschreibt kann nicht allgemein beantwortet werden und die Eignung der Konzepte hängt von der jeweiligen Forschungsfrage ab.

Gegeben den verschiedenen theoretischen Konzepten von Segregation, gibt es eine Vielzahl von Methoden um Segregation zu messen (für einen Überblick z.B. Massey \& Denton, 1988; Reardon \& Firebaugh, 2002; Reardon \& O'Sullivan, 2004; Tivadar, 2019). Dieser Beitrag konzentriert sich auf Konzepte der „evenness“, welche Segregation durch die Ungleichheit der räumlichen Verteilung von Bevölkerungsgruppen messen. Andere Maße beschreiben z.B. Konzepte wie Isolation, räumliche Cluster Bildung, oder Zentralisierung. Das in der Literatur am häufigsten verwendete Maß für Segregation stellt der Dissimilarity Index $(D)$ dar. Im Fall von zwei Gruppen ${ }^{1}$ wird dieser berechnet als:

$$
D=\frac{1}{2} \sum_{i=1}^{N}\left|\frac{t_{i} \pi_{i m}}{T \pi_{m}}-\frac{t_{i}}{T}\right|,
$$

für $i$ räumliche Einheiten, wobei $t_{i}$ : Anzahl Bewohner in Beobachtung $i, T$ : Anzahl Bewohner gesamt, $\pi_{i m}$ : Anteil Bewohner der Gruppe $m$ in Beobachtung $i$ und $\pi_{m}$ : Anteil Bewohner der Gruppe $m$ gesamt. Der Index misst demnach, inwieweit die Proportion der Gruppe $m$ in jeder räumlichen Einheit von der Gesamtproportion der Gruppe $m$ in der Stadt abweicht. Der Index reicht von 0 bis 1 und kann interpretiert werden als Anteil

\footnotetext{
${ }^{1}$ Siehe z.B. Reardon und Firebaugh (2002) für die Erweiterung auf mehrere Gruppen.
} 
der Bewohner von Gruppe $m$, die umziehen müssten um eine gleichmäßige Verteilung über alle Beobachtungen zu erlangen. Der Wert 1 (oder 100\%) gibt folglich das höchste Maß an Segregation an.

Ein zweites Maß, das in der US Literatur mittlerweile dem Dissimilarity Index vorgezogen wird (Reardon \& O'Sullivan, 2004), ist der Theil's Information Theory Index $(H)$. Der Information Theory Index basiert auf dem Entropy Maß

$$
E_{i}=\sum_{m=1}^{M} \pi_{i m} \ln \left(\frac{1}{\pi_{i m}}\right) .
$$

Die Entropy $E$ kann interpretiert werden als ein Maß der Diversität. E nimmt den Wert 0 an, wenn alle Bewohner einer Stadt der Gruppe $m$ angehören und geht gegen 1 wenn alle Bewohner gleichmäßig auf alle $M$ Gruppen aufgeteilt sind. Theil's Information Theory Index ist dann die gewichtete Abweichung der Entropy in jeder räumlichen Einheit $\left(E_{i}\right)$ von der gesamten Entropy der Stadt $(E)$ :

$$
H=\sum_{i=1}^{N} \frac{t_{i}\left(E-E_{i}\right)}{E T} .
$$

Grundsätzlich ist einer der Vorteile des Theils Indizes $H$, dass der Index im Fall von mehreren Gruppen (im Gegensatz zu $D$ ) sensitiv auf selektive Umzüge reagiert. Wenn z.B. eine Person aus einer Gruppe der Minderheiten aus einem Gebiet, in welchem diese Minderheiten überrepräsentiert ist, in ein Gebiet umzieht, in dem diese Gruppe unterrepräsentiert ist, kann es dazu kommen, dass der Multi-Gruppen Index $D$ ansteigt anstatt zu sinken (für Details siehe Reardon \& Firebaugh, 2002). Theils Index $H$ hingegen wäre in diesem Fall strikt abnehmend, was eher der theoretischen Erwartung entspricht. Für den Vergleich von mehr als zwei ethnischen Gruppen, wie in den USA meist üblich, wird $\mathrm{H}$ daher mittlerweile bevorzugt. Im Fall von zwei Gruppen hängt $H$ allerdings, im Gegensatz zu $D$, von der gesamten Proportion der Minderheiten ab und reagiert damit sensitiv auf den Gesamtanteil der Minderheiten innerhalb der Stadt (für eine detaillierten Vergleich siehe z.B. Reardon \& Firebaugh, 2002).

\subsection{Bisherige Befunde}

Obwohl das Thema der räumlichen Segregation in Deutschland bisher weit weniger behandelt wurde als in den USA, gibt es einige gut dokumentierte Befunde in Deutschland. Im internationalen Vergleich scheint das Ausmaß an räumlicher ethnischer Segregation in Deutschland relativ gering zu sein. In einer Meta-Analyse zeigt Musterd (2005), dass Deutschland weit geringere Segregationswerte aufweist als andere europäische Städte. Britische Städte weisen demnach mit einem Segregationsindex von bis zu 0.75 ein sehr hohes Ausmaß an Segregation auf, dicht gefolgt von amerikanischen Städten, die um das Jahr 2000 herum bei ca. 0.65 lagen. Deutsche Städte liegen hingegen mit Segregationswerten zwischen 0.13 (München) und 0.30 (Düsseldorf) am unteren Ende des Vergleich. Auch für verschiedene Einwanderungsgruppen findet Musterd (2012) für den Zeitraum von 1986 bis 2004 in deutschen Großstädten im europäischen Vergleich sehr niedrige Segregationswerte. Basierend auf NUTS3 Regionen in Europa zeigen Lichter, Parisi und Ambinakudige (2019) allerdings, dass Malta, Slowakei, Schweden und Irland die geringsten Segregationswerte in Europa aufweisen, während Deutschland sich eher im unteren Mittelfeld bewegt. Hierbei handelt es sich allerdings um eine sehr groß- 
räumige Form der Segregation. Generell, ist die Vergleichbarkeit zwischen Städten und Regionen in vielen Studien kritisch zu beurteilen, da unterschiedliche räumliche Einheiten mit verschiedenen geographischen Dimensionen verglichen werden.

Was die Entwicklung der Segregation in Deutschland über die Zeit angeht, scheinen bisherige Befunde weniger eindeutig. Auf Grundlage von Microm-Daten auf Ebene von Postleitzahlbezirken und Straßenabschnitten finden Goebel und Hoppe (2015) relativ stabile Segregations- und Isolationswerte für die ausländische Bevölkerung über den Zeitraum von 2005 bis 2013. Unabhängig von der betrachteten Region oder der Größenklasse lassen sich hier kaum Veränderungen über die Zeit erkennen, wobei der Dissimilaritätsindex bei ungefähr 0.15 auf Ebene der Postleitzahl-Bezirke und 0.65 auf Ebene der Straßenabschnitte liegt. Basierend auf amtlichen Daten von Stadtteilen zeigen Helbig und Jähnen (2018) allerdings tendenziell einen leichten Rückgang der ethnischen Segregation. Während der Dissimilationsindex 2002 für alle deutschen Städte zusammen noch bei ca. $0.26 \mathrm{lag}$, findet die Studie 2014 nur noch einen Wert von ca. 0.24, mit einem linear abnehmenden Trend zwischen 2002 und 2014. Auch für Ost und Westdeutschland getrennt finden sich negative Trends im Ausmaß der ethnischen Segregation.

Auch die Ursachen von räumlicher Segregation sind empirisch umstritten. Zum Beispiel zeigt Sager (2012) anhand des SOEP und Microm Daten, dass 2008 ein erheblicher Anteil der individuellen räumlichen Isolation durch Einkommen, Bildung, Sprachkenntnisse und die Größe der Gemeinde erklärt werden kann. Basierend auf Daten zu Postleitzahlbezirken in fünf deutschen Großstädten zeigen Teltemann et al. (2015) allerdings, dass sozioökonomische Faktoren auf der Makro-Ebene nur etwa 20\% der gesamten Segregation erklären können. Zudem zeigen Best und Rüttenauer (2018) und Lersch (2013) für verschiedene Indikatoren der Qualität von Nachbarschaften, dass Haushalte ohne Migrationshintergrund ihre Situation durch Umzüge wesentlich stärker verbessern können als Migranten. Auch hier lässt sich nur ein sehr geringer Anteil der Wohnortswahl durch Einkommen erklären. Im Allgemeinen scheint das Bildungsniveau und das Einkommen zwar einen negativen Einfluss zu haben auf die Wahrscheinlichkeit in Gebieten mit hohem Ausländeranteil zu wohnen, doch lassen sich ethnische Differenzen in der Wohnortswahl dadurch oft nur unzureichend erklären Janßen und Schroedter (2007); Winke (2018).

Oft wird der verbleibende „residuale“ Anteil unterschiedlichen Präferenzen oder Diskriminierung auf dem Wohnungsmarkt zugeschrieben. Tatsächlich, zeigen aktuell Ergebnisse ein vergleichbares (über die Zeit abnehmendes) Maß an Diskriminierung auf dem Wohnungsmarkt in Deutschland und den USA (Auspurg, Schneck \& Hinz, 2019). Ergebnisse in München deuten allerdings auch darauf hin, dass das Ausmaß an Diskriminierung in Gebieten mit hohen Preisen und niedrigem Ausländeranteil tendenziell geringer ist (Auspurg, Hinz \& Schmid, 2017), was räumliche Segregation eher verringern sollte. Generell bleibt die Rolle von Diskriminierung - obwohl unbestritten präsent - als Erklärung für Segregation daher bisher eher unklar. Auch Homophilie, also die Tendenz in Nachbarschaften mit einem hohen Anteil der selben ethnischen Gruppe zu ziehen, liefert eine mögliche Erklärung für Segregation. Diese Verhaltensweise kann vor allem für neu immigrierte Person mit niedrigen Sprachkenntnissen eine schnelle Integration in Netzwerke und den Arbeitsmarkt erleichtern (Logan, Zhang \& Alba, 2002). Winke (2018) zeigt z.B. für Deutschland, dass sich vor allem Immigranten der ersten Generation in Gebieten mit hohem Anteil ethnischer Minderheiten bewegen, während in der zweiten Generation keine Unterschiede mehr zu Personen ohne Migrationshintergrund zu finden sind.

Relativ wenige empirische Studien betrachten bisher die Struktur der ethnischen Segregation in Deutschland. Die Ergebnisse von Goebel und Hoppe (2015) und Helbig 
und Jähnen (2018) lassen tendenziell darauf schließen, dass das Level an Segregation im Osten wesentlich höher ist als im Westen. Außerdem zeigt die Studie von Helbig und Jähnen (2018), dass türkischstämmige Migranten in den 51 Städten der Innerstädtische Raumbeobachtung (IRB, siehe z.B. Jähnen \& Helbig in diesem Band) die am stärksten segregierte Gruppe ist. Frühere Ergebnisse (Musterd, 2012) deuten allerdings darauf hin, dass Schlussfolgerungen bezüglich einzelner Gruppen stark zwischen Städten variieren. Eine weitere Beobachtung machen Friedrichs und Triemer (2009) in ihrem Vergleich deutscher Großstädte: In (fast) allen deutschen Städten ist der Ausländeranteil in einem Kerngebiet von wenigen Bezirken besonders hoch und nimmt von dort aus kontinuierlich ab. Es scheint also in deutschen Großstädten geclusterte Gebiete mit hohem Ausländeranteil (meist relativ zentral) und periphere Gebiete mit niedrigem Anteil zu geben. Für ethnische Minderheiten in den USA zeigen Studien ähnliche Befunde. Hier hat die "Ghettoisierung" der Schwarzen Bevölkerung zu einem relative hohen Anteil im Stadtkern und niedrigen Anteilen in der Peripherie geführt (Logan et al., 2002).

\subsection{Die räumliche Struktur von Segregation}

Obwohl die obigen Studien wichtige Erkenntnisse über das Ausmaß und einige strukturelle Eigenschaften der Segregation liefern, vermeiden die Studien es, Vergleiche über das absolute Ausmaß über Städte hinweg anzustellen. Das liegt vor allem daran, dass herkömmliche Segregationsindizes basierend auf amtlichen Einwohnerstatistiken eine Reihe von Problemen aufweisen: 1) Messungen basierend auf unterschiedlich räumlich eingeteilten Daten und sind damit nicht vergleichbar, 2) herkömmliche Indizes vernachlässigen die räumliche Dimension von Segregation, und 3) Segregation kann auf unterschiedlichen Ebenen auftreten.

Das erste Problem der Vergleichbarkeit über die Städte hinweg ist verwandt mit dem allgemeinen Problem von arbiträren Grenzen in räumlichen Analysen, dem Modifiable Areal Unit Problem (MAUP; Wong, 2009). Die Einteilung der Bevölkerung in verschiedene geographische Bereiche erzeugt dabei zwei methodische Probleme.

Zum einen kann die räumliche Größe der einzelnen Bereiche relativ stark variieren. Da herkömmliche Segregationsindizes den Anteil an Minderheiten in jedem einzelnen geographischen Bereich mit dem Anteil an Minderheiten im gesamten Stadtgebiet vergleichen, erzeugen unterschiedliche Größen ein Problem (Lee et al., 2008). Vergleicht man zum Beispiel die selbe Stadt, einmal eingeteilt in zwei Stadtteile und einmal eingeteilt in 100 Bezirke, werden die Anteile an Minderheiten auf der Ebene von 100 Bezirken wesentlich stärker von der Gesamtzusammensetzung der Stadt abweichen. Auf der höheren Ebene von zwei Stadtteilen hingegen werden alle kleinräumigen Unterschiede verdeckt und die Wahrscheinlichkeit ist hoch, dass beide Stadtteile eine relativ ähnliche Zusammensetzung aufweisen wie die gesamte Stadt. Herkömmliche Segregationsindizes sind daher „skalenvariant“ (Reardon \& O'Sullivan, 2004) und können nicht über Stadtgrenzen hinweg verglichen werden, solange diese nicht gleichermaßen unterteilt sind. Zum anderen hängt das Ausmaß an Segregation mit der Grenzziehung selbst zusammen. Wenn z.B. die administrativen Grenzen von Nachbarschaften entlang von ethnischen Clustern gezogen werden, ergeben herkömmliche Indizes einen höheren Wert als im Falle von Grenzen, die durch diese Cluster hindurch gezogen werden. Damit hängen die Ergebnisse von den Grenzen der Untergliederungseinheiten ab und die Indizes würden sich verändern, wenn wir die Grenzen leicht verschieben würden.

Das zweite Problem der Vernachlässigung der räumlichen Dimension hängt damit zusammen, dass herkömmliche Indizes zwar Unterschiede in der Zusammensetzung von 


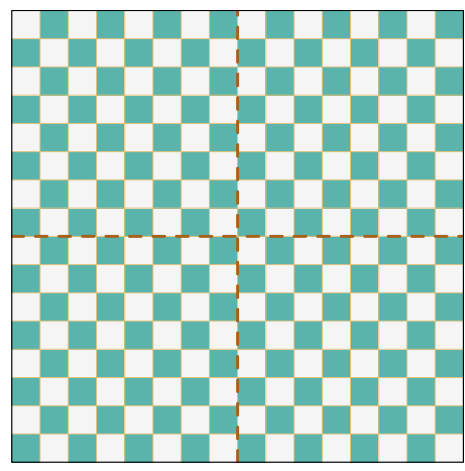

a)

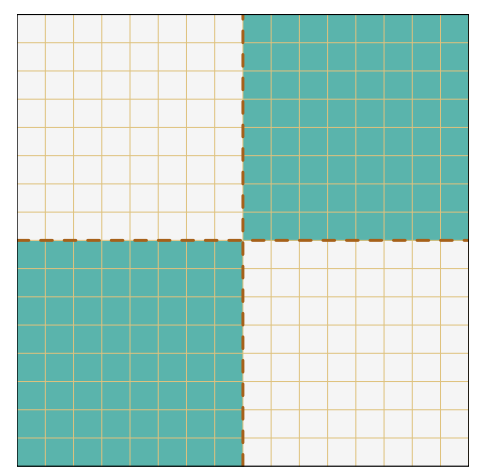

b)

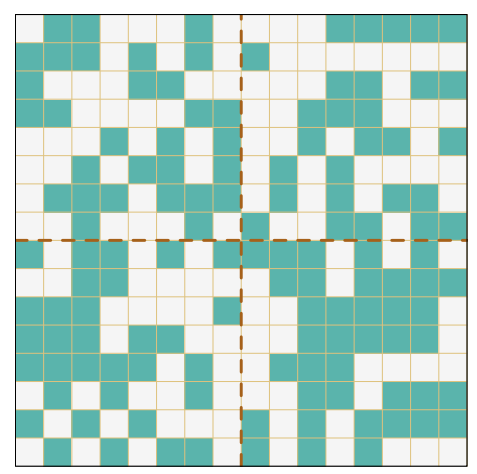

c)

Abbildung 1. Varianten der räumlichen Struktur von Segregation

Stadtteilen berechnen, dabei aber nicht berücksichtigen, wo diese Stadtteile geographisch liegen (Lee et al., 2008; Reardon et al., 2008; Reardon \& O'Sullivan, 2004). Das Schachbrett in Abbildung 1 stellt die Problematik beispielhaft dar (Duncan \& Duncan, 1955). Eine Stadt ist aufgeteilt in $16 \times 16$ Nachbarschaften (z.B. Straßenblöcke) und wir nehmen an, dass dunkle Kästchen einen niedrigen Anteil an Minderheiten und helle Kästchen einen hohen Anteil an Minderheiten bedeuten. Die gestrichelten Linien teilen die hypothetische Stadt in vier größere Stadtteile ein. In Abbildung 1 a) sind Regionen mit einem hohen Anteil an Minderheiten - wie in einem Schachbrett Muster - gleichmäßig über das gesamte Gebiet verteilt. In Abbildung 1 b) hingegen sind alle Einheiten mit hohem Anteil an Minderheiten (helle Kästchen) auf zwei größere Bereiche verteilt und alle mit niedrigem Anteil befinden sich in zwei anderen größeren Bereichen. Abbildung 1 c) hingegen ist eine zufällige Verteilung mit einigen kleineren Clustern beider Gruppen.

Vergleicht man die drei Muster sieht man sofort, dass es in Szenario a) für die Gruppe der Minderheit wesentlich leichter ist mit Einwohner der anderen Gruppe in Kontakt zu kommen. Auch in Szenario c) sind die Distanzen für viele räumliche Positionen zwischen den beiden Gruppen noch nicht sonderlich hoch, wenn auch manche Wohnorte etwas stärker abgeschottet sind als andere. In Szenario b) hingegen gibt es viele Nachbarschaften, die absolut abgegrenzt sind vom anderen Typ der Nachbarschaften, also viele Bewohner die umgeben sind von einer homogenen Zusammensetzung der Gebiete. Personen im Kästchen ganz unten rechts haben zum Beispiel einen weiten geographischen Weg um in das nächste Kästchen mit einem niedrigen Anteil an Minderheiten zu kommen (unten Mitte oder Mitte rechts). Auch für die subjektive Wahrnehmung von ethnischen Unterschieden innerhalb einer Stadt mag die geclusterte Segregation in Szenario b) eine stärkere Rolle spielen als die Muster in a) und c), da sich ganze Stadtteile qualitativ in der ethnischen Zusammensetzung unterscheiden. Herkömmliche Segregationsmaße können diese Unterschiede allerdings nicht abbilden, da nur die Verteilung in jeder räumlichen Einheit, nicht aber deren räumliche Position betrachtet wird. Herkömmliche Segregationsindizes würden für alle drei Szenarien - a), b) und c) - den selben Wert an Segregation ergeben, da diese Maße davon ausgehen, dass alle Gebiete gleich weit voneinander entfernt sind und Bewohner keinen Kontakt zu Personen über die Grenzen der administrativen Einheiten hinaus haben (Lee et al., 2008).

Das dritte Problem nicht-räumlicher Segregationsindizes betrifft im Grunde eine theoretische Fragestellung, die aus der obigen Unterscheidung folgt: Auf welcher Ebene 
wollen wir Segregation messen? Werden die administrativen Einheiten verwendet, auf deren Ebene die Daten zur Verfügung stehen, nimmt man implizit an, dass diese Einheiten den korrekten Maßstab für eine "Nachbarschaft" abbilden (Lee et al., 2008). Es ist aber denkbar, dass Segregation auf verschiedenen geographischen Ebenen stattfindet. Für die USA unterscheiden Lichter, Parisi und Taquino (2015) zwischen Mikro- und Makro-Segregation. Mikro-Segregation stellt dabei die räumliche Aufteilung innerhalb von Stadtteilen dar, also die Abgrenzung auf kleinräumiger Ebene. Gehen wir davon aus, Abbildung 1 ist durch die gestrichelten Linien in vier große Stadtteile unterteilt, dann würde Szenario a) eine hohe Mikro-Segregation innerhalb der vier Stadtteile aufweisen. Makro-Segregation hingegen stellt eine Trennung von ethnischen Gruppen auf der Ebene von Stadtteilen dar. So ist es z.B. möglich, dass ein Großteil der Minderheiten zwar gleichmäßig um den Stadtkern herum verteilt lebt (also innerhalb des Zentrums wenig segregiert ist), Vororte oder suburbane Gebiete hingegen aber alle einen sehr geringen Anteil an Minderheiten aufweisen. In diesem Fall wären die Gruppen auf einer großräumigen Ebene voneinander getrennt, eine Situation die Szenario b) entspricht. Betrachten man hingegen Szenario a) auf Ebene der vier Stadtteile, so haben alle Stadtteile den selben Anteil an Minderheiten und die Makro-Segregation wäre 0 (perfekte Gleichverteilung über die vier Stadtteile).

Diese Unterscheidung ist daher wichtig, da bisherige Forschung darauf hindeutet, dass Mikro- und Makro-Segregation zwei unterschiedliche Prozesse der räumlichen Trennung darstellen. Lichter et al. (2015) finden in den USA zwischen 1990 und 2010 zwar eine abnehmende Mikro-Segregation, gleichzeitig aber auch eine zunehmende Segregation zwischen den Stadtteilen (Makro-Segregation). Außerdem ist davon auszugehen, dass beide Arten der Segregation wahrscheinlich von unterschiedlichen Prozessen getrieben werden (Lee et al., 2008). Strukturelle Merkmale, wie der absolute Anteil an Minderheiten oder der Bevölkerungsanstieg in Städten (z.B. Teltemann et al., 2015), haben vermutlich stärkere Auswirkungen auf die Makro Komponente, da sie einen stärkeren Einfluss auf die Gesamtzusammensetzung und Cluster-Bildung zwischen Stadtteilen haben (Lee et al., 2008). Infrastruktur oder die geographische Verteilung von institutionellen Einrichtungen hingegen könnten stärkere Auswirkungen auf die Mikro-Segregation haben, da diese Infrastrukturen natürliche Barrieren darstellen und so abrupte Brüche in der demographischen Zusammensetzung entstehen können (Reardon et al., 2008; Roberto, 2018). Ebenso könnten beide Varianten der Segregation unterschiedliche Folgen für die Bewohner haben. So ist die Mikro-Segregation wahrscheinlich stärker im alltäglichen Umfeld von Bewohnern wahrnehmbar, während die Makro-Segregation eher zu einer Trennung des gesamten Stadtbildes führt und damit z.B. einen stärkeren Einfluss die Stigmatisierung gesamter Stadtteile hat. Für viele sozialwissenschaftliche Fragestellungen ist es daher von Bedeutung, Segregation auf verschiedenen geographischen Ebenen zu betrachten und das theoretisch geeignete Maß zu verwenden: „one size does not fit all“ (Lee et al., 2008, p. 769).

\section{Räumliche Segregationsindizes}

Um die oben beschriebenen Probleme zu lösen schlagen Reardon und O'Sullivan (2004) eine Reihe von räumlichen Segregationsmaßen vor (siehe auch Lee et al., 2008; Reardon et al., 2008), welche explizit die räumliche Verteilung der einzelnen geographischen Einheiten berücksichtigen. Hierzu wird die Verteilung der Bevölkerung an verschiedenen geographischen Punkten $p$ in der Region der Stadt verwendet um anhand der lokalen Zusammensetzung $\tilde{\pi}_{p m}$ Segregation zu messen. Der räumliche Dissimilarity Index $(\tilde{D})$ 
wird demnach berechnet als

$$
\tilde{D}=\sum_{m=1}^{M} \int_{p \in R} \frac{\tau_{p}}{2 T I}\left|\tilde{\pi}_{p m}-\pi_{m}\right| d p,
$$

und der räumliche Theil's Information Index $(\tilde{H})$ wird berechnet als

$$
\tilde{H}=1-\frac{1}{T E} \int_{p \in R} \tau_{p}\left[-\sum_{m=1}^{M}\left(\tilde{\pi}_{p m}\right) \log _{M}\left(\tilde{\pi}_{p m}\right)\right] d p
$$

wobei jeweils:

$\tau_{p}$ : Bevölkerungsdichte (Anzahl / Fläche) an Punkt $p$,

$\tau_{p m}$ : Bevölkerungsdichte der Gruppe $m$ an Punkt $p$,

$\pi_{m}$ : gesamter Anteil der Gruppe $m$,

$\tilde{\pi}_{p m}$ : Anteil der Gruppe $m$ in der lokalen Nachbarschaft von $p$,

$T$ : gesamte Bevölkerung,

$I=\sum_{m=1}^{M}\left(\pi_{m}\right)\left(1-\pi_{m}\right)$,

$E=-\sum_{m=1}^{M}\left(\pi_{m}\right) \log _{M}\left(\pi_{m}\right)$.

Diese räumlichen Indikatoren haben den Vorteil, dass das Ausmaß an Segregation für verschiedene Definitionen von Nachbarschaften berechnet werden kann. Die lokale Nachbarschaft kann z.B. als die Region in einem Radius von $100 \mathrm{~m}$ oder von $2 \mathrm{~km}$ um jeden Punkt $p$ definiert werden. Anschließend werden die Abweichungen der Anteile jeder Gruppe in diesen lokalen Nachbarschaften vom Gesamtanteil innerhalb der Stadt berechnet. Dadurch kann zum einen vorweg explizit definiert werden, welche Größe jede Nachbarschaft hat und zum andern kann das Ausmaß an Segregation auf verschiedenen räumlichen Skalen gemessen werden. Praktisch werden für die Schätzung dieser räumlichen Indizes Dichte Funktionen verwendet um die Verteilung basierend auf den vorhandenen Daten zu schätzen. In Abbildung 2 ist eine Schätzung der Berliner Bevölkerung basierend auf Kernel Dichte Schätzern dargestellt. Während im oberen Bereich eine Bandbreite von 100m verwendet wird, zeigt der untere Teil die Schätzung anhand einer $2 \mathrm{~km}$ Bandbreite. Dadurch lassen sich im ersten Beispiel Muster von Mikro-Segregation und zweiten Beispiel Muster von Makro-Segregation erkennen, die unabhängig von der räumlichen Einteilung der ursprünglichen Daten sind.

Diese räumlichen Indikatoren der Segregation addressieren die zuvor genannten Kritikpunkte an konventionellen Segregationsindizes. Für die Schätzung der Indizes werden räumliche Dichte Funktionen verwendet um die Verteilung basierend auf den vorhandenen Daten zu schätzen. In Abbildung 2 ist eine Schätzung der Berliner Bevölkerung basierend auf Kernel Dichte Schätzern dargestellt. Während im oberen Bereich eine Bandbreite von 100m verwendet wird, zeigt der untere Teil die Schätzung anhand einer $2 \mathrm{~km}$ Bandbreite. Gegeben die ursprünglichen Daten haben eine feine räumliche Auslösung, werden die Analyse-Daten dadurch unabhängig von der ursprünglichen Einteilung der Daten (Problem 1). Außerdem wird für die ethnische Zusammensetzung jedes Punktes $\mathrm{p}$ in der Stadt nicht nur die Bevölkerung in der jeweiligen Einheit, sondern auch die Bevölkerungszusammensetzung in umliegenden Einheiten berücksichtigt. Damit gehen unterschiedliche räumliche Muster und Distanzen wie in Abbildung 1 in die Berechnung mit ein (Problem 2). Wie in Abbildung 2 zu sehen, kann eine lokale Nachbarschaft außerdem durch verschiedene Radii definiert werden, wie hier z.B. mit einem Radius von $100 \mathrm{~m}$ oder von $2 \mathrm{~km}$ um jeden Punkt $p$ (siehe z.B. auch Schaeffer, Jünger \& Klin- 
Lokale Nachbarschaft $=100 \mathrm{~m}$

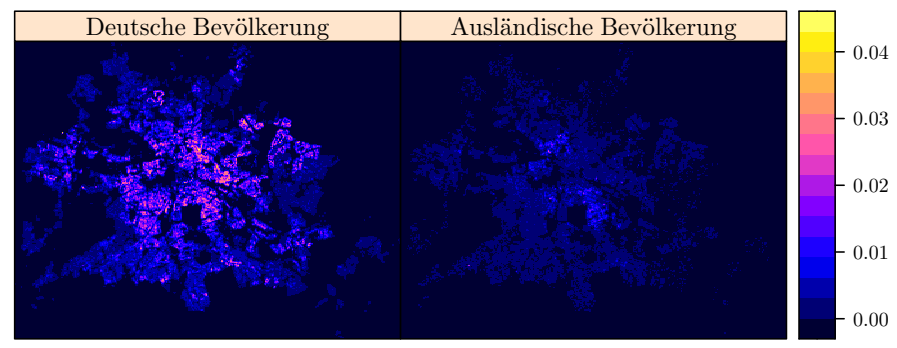

Lokale Nachbarschaft $=2 \mathrm{~km}$

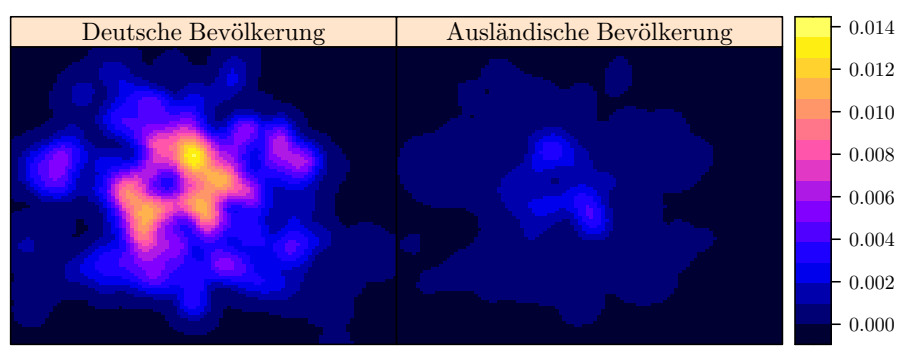

Abbildung 2. Verschiedene Bandbreiten der lokalen Nachbarschaft für räumliche Segregationsmaße am Beispiel Berlin

ger in diesem Band). Dadurch kann Segregation auf verschiedenen räumlichen Ebenen gemessen werden (Problem 3). Ähnlich den konventionellen Indizes, werden auch hier die Abweichungen der Anteile jeder Gruppe in diesen lokalen Nachbarschaften vom Gesamtanteil innerhalb der Stadt berechnet. So lassen sich Muster von Mikro-Segregation (Abb. 2 oben) und Muster von Makro-Segregation (Abb. 2 unten) erkennen, die unabhängig von der räumlichen Einteilung der ursprünglichen Daten sind.

Wie im Falle der nicht-räumlichen Schätzer variieren auch die räumlichen Indizes zwischen Werten von 0 (minimale Segregation) und 1 (maximale Segregation). Wichtig ist dabei, dass das Ausmaß an Segregation immer eine nicht-ansteigende Funktion der verwendeten Bandbreite ist (Reardon et al., 2008). Je kleiner die Bandbreite, desto näher kommt die Definition der lokalen Nachbarschaften theoretisch an einzelne Haushalte heran, welche sich erwartungsgemäß (da oft homogen) maximal von der Zusammensetzung der gesamten Stadt unterscheiden. Je größer die Bandbreite, desto näher kommt die Definition der Nachbarschaft an die gesamte Stadt heran, welche dann per Definition der gesamten Verteilung innerhalb der Stadt entspricht. Für die Berechnungen der Segregationsindizes wird in dieser Studie das $R$ Paket seg verwendet (Hong, O'Sullivan \& Sadahiro, 2014).

Obwohl die beiden oben genannten Konzepte die herkömmlichen Indizes entscheidend erweitern, stellen die beiden räumlichen Indizes nur einen Bruchteil der möglichen Maße dar (für einen aktuellen Überblick siehe z.B. Tivadar, 2019). Je nach theoretischer Fragestellung können auch andere Operationalisierungen von Segregation passend sein, die weit über die hier dargestellten Maße hinausgehen. Für die ungleiche Belastung durch Luftverschmutzung z.B. ist die Zentralität von größerer Bedeutung als die allgemeine ungleiche Verteilung (Rüttenauer, 2019a), während für Konflikte eher geographische Übergänge wichtig sind (Klinger, Müller \& Schaeffer, 2017; Legewie \& Schaeffer, 2016). Neuere Methoden versuchen außerdem Konzepte wie Wegstrecken (Roberto, 2018), oder Pendelströme (Phillips, Levy, Sampson, Small \& Wang, 2019) in die 
Tabelle 1. Deskriptive Statistiken.

\begin{tabular}{|c|c|c|c|c|c|c|c|c|}
\hline \multirow[b]{2}{*}{ Variable } & \multicolumn{4}{|c|}{ Westdeutschland } & \multicolumn{4}{|c|}{ Ostdeutschland } \\
\hline & Mean & $\mathrm{SD}$ & Min & Max & Mean & $\mathrm{SD}$ & Min & Max \\
\hline Einwohner & 66.64 & 58.88 & 3.0 & 1702.0 & 78.91 & 79.63 & 3.0 & 1113.0 \\
\hline \% Ausländer & 9.79 & 12.15 & 0.0 & 100.0 & 4.74 & 8.68 & 0.0 & 100.0 \\
\hline$\%$ Immigranten & 15.98 & 14.29 & 0.0 & 100.0 & 8.10 & 10.68 & 0.0 & 100.0 \\
\hline \% Südeuropa & 0.99 & 3.19 & 0.0 & 100.0 & 0.15 & 1.04 & 0.0 & 100.0 \\
\hline \% Osteuropa & 1.28 & 3.62 & 0.0 & 100.0 & 0.75 & 2.49 & 0.0 & 100.0 \\
\hline \% Türkei & 2.34 & 5.82 & 0.0 & 100.0 & 0.81 & 3.17 & 0.0 & 100.0 \\
\hline $\mathrm{N}$ obs. & \multicolumn{4}{|c|}{278048} & \multicolumn{4}{|c|}{71407} \\
\hline
\end{tabular}

Messung räumlicher Segregation zu integrieren. Für viele Fragestellungen ist es daher wichtig, die richtige Auswahl an theoretisch relevanten Dimensionen der Segregation zu treffen.

\section{Daten}

Um das Ausmaß und die Struktur der Segregation über deutsche Großstädte hinweg zu vergleichen, greift dieser Beitrag auf Daten des Zensus 2011 zurück (Statistische Ämter des Bundes und der Länder, 2015). Der Zensus stellt eine Schätzung der gesamten in Deutschland lebenden Bevölkerung basierend auf bereits vorhandenen Melderegister-Daten und ergänzenden Befragungen (ca. 10\% der Bevölkerung) im Mai 2011 dar. Demographische Merkmale werden dabei auf der räumliche Ebene von 100m $\times 100 \mathrm{~m}$ großen Gitterzellen über Deutschland zur Verfügung gestellt, was eine sehr genaue räumliche Gliederung erlaubt. Insgesamt werden Information für 1,855,939 Gitterzellen mit mindestens 3 Bewohnern zur Verfügung gestellt.

Da es sich bei Segregation um ein städtisches Phänomen handelt, werden für die folgenden Analysen nur jene Gitterzellen beachtet, die basierend auf dem Stand 2011 in einer der 79 deutschen Großstädte ( $\geq 100.000$ Einwohner) liegen. Damit reduziert sich der finale Datensatz für die folgenden Analysen auf 349,455 Gitterzellen. Insgesamt enthalten diese Gitterzellen Informationen zu 24,163,586 Einwohnern mit einer durchschnittlichen Anzahl von 69 Personen pro Gitterzelle.

Der Zensus 2011 enthält sowohl Informationen zur Staatsangehörigkeit als auch zum Geburtsland von Einwohnern jeder Gitterzelle. Dadurch ist es auf dieser Datengrundlage möglich, die Segregation für verschiedene Kategorien von ethnischen Minderheiten zu messen. Der Anteil der ausländischen Bevölkerung, definiert als Personen ohne deutsche Staatsangehörigkeit, ist in bisherigen Studien die am häufigsten verwendete Angabe. Neben dem Ausländeranteil wird in dieser Studie auch die Segregation für Personen mit Migrationshintergrund berechnet, also für Personen, die außerhalb Deutschlands geboren wurden (unabhängig von der Staatsangehörigkeit). Für ein noch feineres Bild, werden außerdem noch getrennte Segregationsmaße für verschiedene Staatsangehörigkeiten berechnet. Um eine genügend große Fallzahl zu gewährleisten, wurden diese in den Gruppen Südeuropa, Osteuropa und Türkei zusammengefasst. Die deskriptiven Statistiken sind in Tabelle $1 \mathrm{zu}$ sehen.

Über Deutschland hinweg weisen die Gitterzellen im Durchschnitt einen Ausländeranteil von 8,76\% auf. Auf Grund der überproportionalen Verteilung von Personen mit ausländischer Staatsbürgerschaft auf bevölkerungsstarke Zellen, entspricht dies einem Anteil von 12,43\% an den ca. 24 Mio Einwohnern der deutschen Großstädte. Unabhängig von der Staatsbürgerschaft, haben ca. 19,10\% der Einwohner einen 


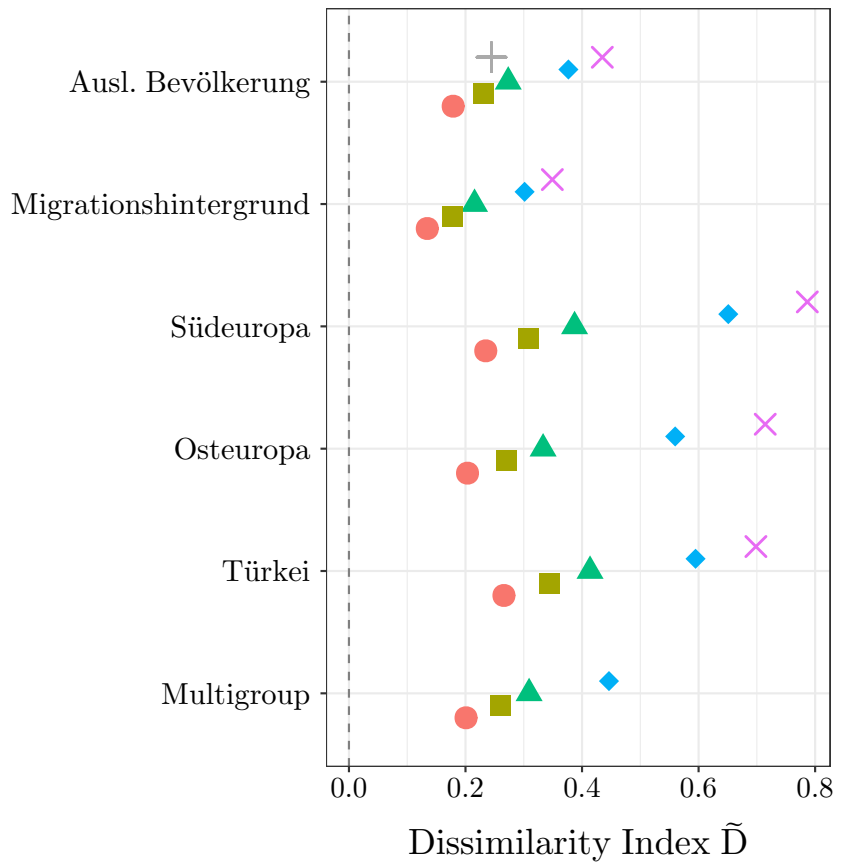

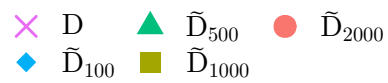

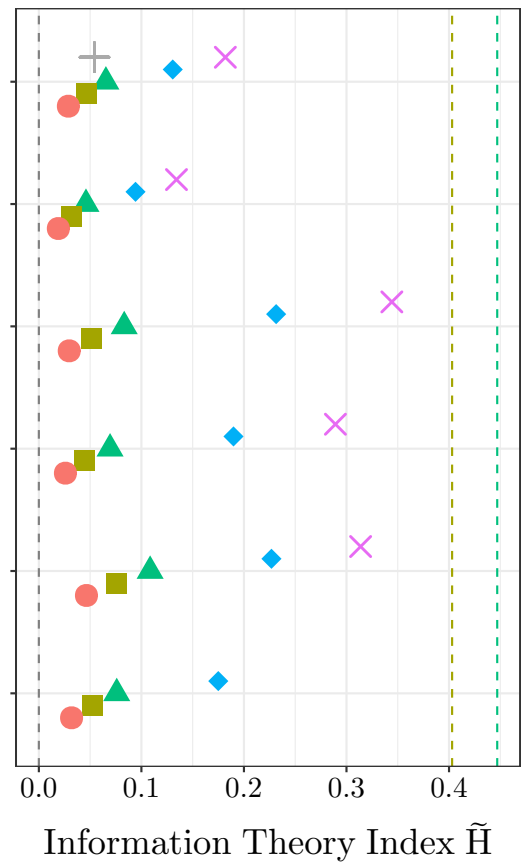

$\times \mathrm{H} \quad \Delta \widetilde{\mathrm{H}}_{500} \bigcirc \widetilde{\mathrm{H}}_{2000}$

Abbildung 3. Durchschnittliches Segregationsmaße für alle 79 Großstädte auf verschiedenen räumlichen Skalen.

Migrationshintergrund (im Ausland geboren), was einem ungewichteten Mittel von 12,43\% pro Gitterzelle entspricht. Insgesamt liegt der Ausländeranteil und der Anteil an Personen mit Migrationshintergrund damit in den deutschen Großstädten deutlich über dem Gesamtanteil 2011 von 7,36\% für die ausländische Bevölkerung und 14,75\% für die Bevölkerung mit Migrationserfahrung. Obwohl der Anteil an ausländischer Bevölkerung in Ostdeutschland nur etwa der Hälfte des Anteils in Westdeutschland entspricht, gibt es in beiden Regionen eine starke Heterogenität: Sowohl in Ost als auch in West schwanken Ausländeranteil und Anteil an Personen mit Migrationserfahrung zwischen 0 und $100 \%$ innerhalb der $100 \mathrm{~m} \times 100 \mathrm{~m}$ Gitterzellen.

\section{Ergebnisse}

In Abbildung 3 sind die durchschnittlichen Segregationsindizes für verschiedene räumliche Skalen über alle 79 deutschen Großstädte hinweg zu sehen (entsprechende Zahlen sind in Tabelle A1 des Appendix zu sehen). Für die Gruppe der ausländischen Bevölkerung weisen die deutschen Großstädte im Durchschnitt einen Dissimilarity Index zwischen $0.18(2 \mathrm{~km})$ und $0.38(100 \mathrm{~m})$ auf. Das bedeutet, dass lokale Nachbarschaften sich auf der großräumigen Ebene $(2 \mathrm{~km})$ nur schwach und auf der kleinräumigen Ebene $(100 \mathrm{~m})$ mittel-stark von der gesamten Zusammensetzung der jeweiligen Stadt unterscheiden. Für Personen, die außerhalb Deutschlands geboren sind - unabhängig von der Staatsbürgerschaft - ist das Ausmaß an Segregation geringer (0.13 bis 0.30). Unterteilt man hingegen die Gruppe der ausländischen Bevölkerung weiter in einzelne Staatsangehörigkeiten und vergleicht die räumliche Verteilung mit der deutschen Bevölkerung, 
ergeben sich wieder höhere Segregationswerte. Interessant ist hier vor allem, dass Personen aus Südeuropa auf einer kleinräumigen Ebene (100m Nachbarschaft) am stärksten segregiert leben, während Personen aus der Türkei am stärksten auf einer großräumigen Skala $(2 \mathrm{~km})$ segregiert von Personen mit deutscher Staatsangehörigkeit leben.

Berechnet man den Quotienten zwischen Makro- und Mikro-Segregation (Reardon et al., 2008), zeigt sich, dass sich für Personen aus Südeuropa ca. 36\% der Mikro-Segregation durch die Makro-Segregation erklären lässt. Für Personen mit türkischer Nationalität lassen sich hingegen $45 \%$ durch großflächige Muster erklären. Inhaltlich bedeuted dies, dass türkischstämmige Einwohner generell stärker auf einer großfächigen Ebene, wie z.B. auf Ebene von Stadtteilen, von Einwohner mit deutscher Staatsbürgerschaft getrennt wohnen als andere Gruppen. Bewohner südeuropäischer Herkunft im Gegensatz leben eher stärker abgegrenzt in kleinräumigen Gebieten (Blöcke oder Straßenabschnitte). Werden alle Nationalitäten gemeinsam für die Berechnung des Multigroup-Indizes herangezogen, liegt das Ausmaß an Segregation zwar unter den Werten für einzelne Gruppen, allerdings auch über dem Ausmaß für den Vergleich zwischen der deutschen und der zusammengefassten ausländischen Bevölkerung ( 0,20 für $2 \mathrm{~km}$ und 0,45 für 100m). Es scheint also auch innerhalb der ausländischen Bevölkerung noch eine räumliche Separierung nach Herkunftsregionen zu geben.

Als Vergleichswert zu bisherigen Ergebnissen markiert das graue "+ " den durchschnittlichen nicht-räumlichen Dissimilarity Index basierend auf den Stadtteilen der 51 IRB Städte (vergleiche Helbig \& Jähnen, 2018) für das Jahr 2011. Mit einem Wert von 0.245 liegt das Ausmaß an Segregation, das wir basierend auf Informationen zu einzelnen Stadtteilen erhalten würden, damit zwischen den räumlichen Indizes für Nachbarschaften mit $500 \mathrm{~m}$ und $1 \mathrm{~km}$ Bandbreite. Tendenziell wird dadurch basierend auf Stadtteilen eher eine Art der Makro-Segregation gemessen, während kleinräumige geographische Unterschiede durch die Größe der Stadtteile überdeckt werden. Vergleicht man die Rangordnung der Städte im Ausmaß an Segregation, so ähnelt die Rangordnung basierend auf Daten der IRB Stadtteile tendenziell der Rangordnung basierend auf den beiden großräumigen Definitionen von Nachbarschaften mit $1 \mathrm{~km}$ oder $2 \mathrm{~km}$ Bandbreite (siehe Tabelle A2). Obwohl der herkömmliche Dissimilarity Index die geographische Position der Stadtteile nicht berücksichtigt, scheint er trotzdem eine relativ gute Annäherung an das Maß der großräumigen Segregation zu liefern.

Betrachtet man statt des Dissimilarity Indizes den Information Theory Index $\tilde{H}$, bleiben die Muster grundsätzlich unverändert. Allerdings ändert sich das generelle Ausmaß der Segregation stark. Für die Gruppe der ausländischen Bevölkerung schwankt $\tilde{H}$ zwischen $0.03(2 \mathrm{~km})$ und $0.13(100 \mathrm{~m})$. Betrachtet man also $100 \mathrm{~m}$ als Größe der Nachbarschaft, leben Personen ohne deutsche Staatsbürgerschaft im Durchschnitt in lokalen Nachbarschaften, die 13\% weniger divers sind als die gesamte Stadt. Damit liegen die Segregationsmaße deutlich unter dem Ausmaß in den USA. Die gestrichelten Linien in Abbildung 3 geben die Resultate für $\tilde{H}_{500}$ und $\tilde{H}_{1000}$ aus den USA nach Lee et al. (2008) wieder. Für eine lokale Umgebung von 500m messen Lee et al. (2008) einen Index von 0.45 (0.40 für 1km) für den Vergleich zwischen Schwarzen und Weißen Bevölkerungsgruppen, wohingegen deutsche Großstädte einen Index von 0.07 (bzw. 0.05) für ausländische Minderheiten aufweisen. Damit ist das Ausmaß an Segregation in Deutschland deutlich niedriger als in den USA. Allerdings ist dieser Vergleich mit Vorsicht zu interpretieren, da $\tilde{H}$ von der absoluten Größe der Minderheit abhängt.

Über die deutschen Städte hinweg, zeigt der Dissimilarity Index $\tilde{D}$ eine beachtliche Heterogenität zwischen den Städten: Tabellen A2 und A3 des Appendix zeigen die Werte für alle Städte. In Abbildung 4 sind die räumlichen Unterschiede grafisch für ausländische Staatsbürger allgemein und die Gruppe der türkischen Minderheiten darge- 


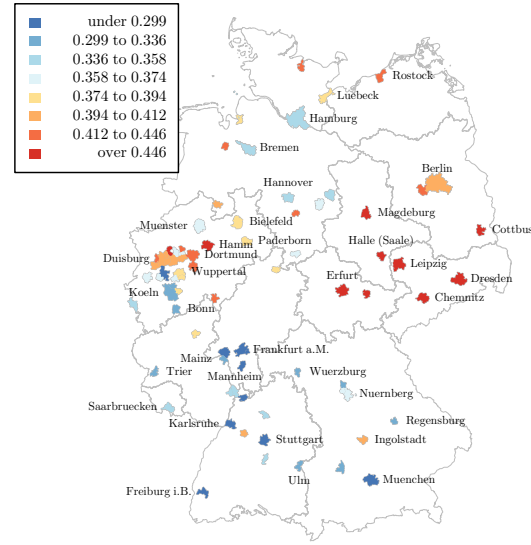

$\widetilde{\mathrm{D}}_{100}$ Tuerkische Bevoelkerung

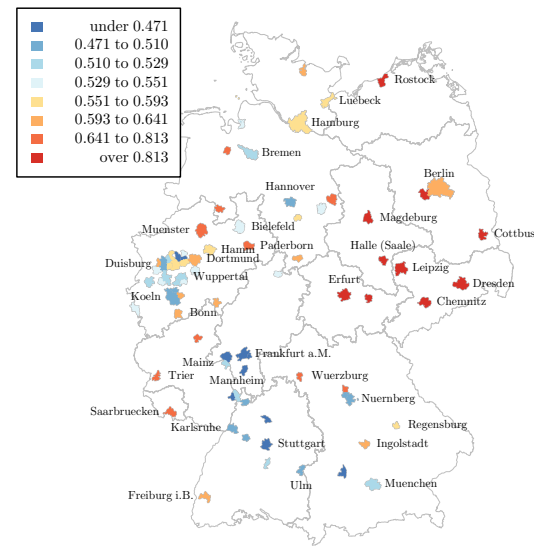

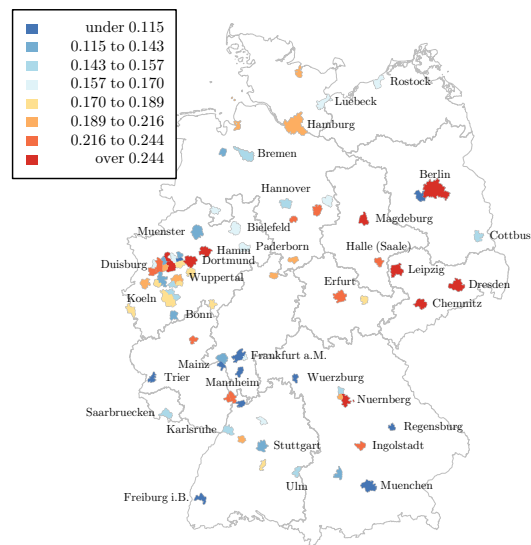

$\widetilde{\mathrm{D}}_{2000}$ Tuerkische Bevoelkerung

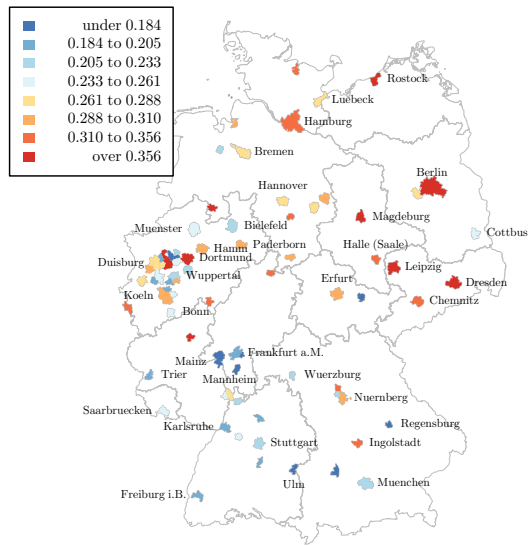

Abbildung 4. Das räumliche Ausmaß an Segregation für ausgewählte lokale Bandbreiten von $\tilde{D}$.

stellt. Besonders auffällig ist dabei, dass das Ausmaß an Segregation - über alle Ebenen hinweg - besonders hoch im Osten und besonders niedrig im Süden und Süd-Westen (südliche Rhein-Region, Baden-Württemberg und Bayern) ist. Für eine lokale Nachbarschaft von 100m weisen Chemnitz und Erfurt (0.56 und 0.52) das höchste Level an Segregation für ausländische Minderheiten auf. Frankfurt am Main und Heidelberg hingegen sind die am wenigsten segregierten Städte (jeweils 0.24). Auf der Makro Ebene $(2 \mathrm{~km})$ ist die Segregation in Chemnitz und Berlin am höchsten (0.32 und 0.31) und in Heidelberg und Potsdam am niedrigsten (0.08 und 0.09). Ein ähnliches geographisches Bild zeigt sich auch für die türkischen Minderheiten. Kleinräumig ergeben sich vor allem in der Metropolregion Rhein-Ruhr auffällige Unterschiede. Die Mikro-Segregation ist z.B. in Hamm und Bottrop mit 0.47 und 0.46 sehr hoch, wohingegen Gelsenkirchen mit 0.37 im Mittelfeld liegt und Düsseldorf mit 0.29 eine eher geringe Segregation aufweist. Auch auf der Makro-Ebene von $2 \mathrm{~km}$ wiederum weist Düsseldorf sehr niedrige Werte (0.13) auf, wohingegen Gelsenkirchen eine eher stark ausgeprägte Segregation (0.29) zeigt. 


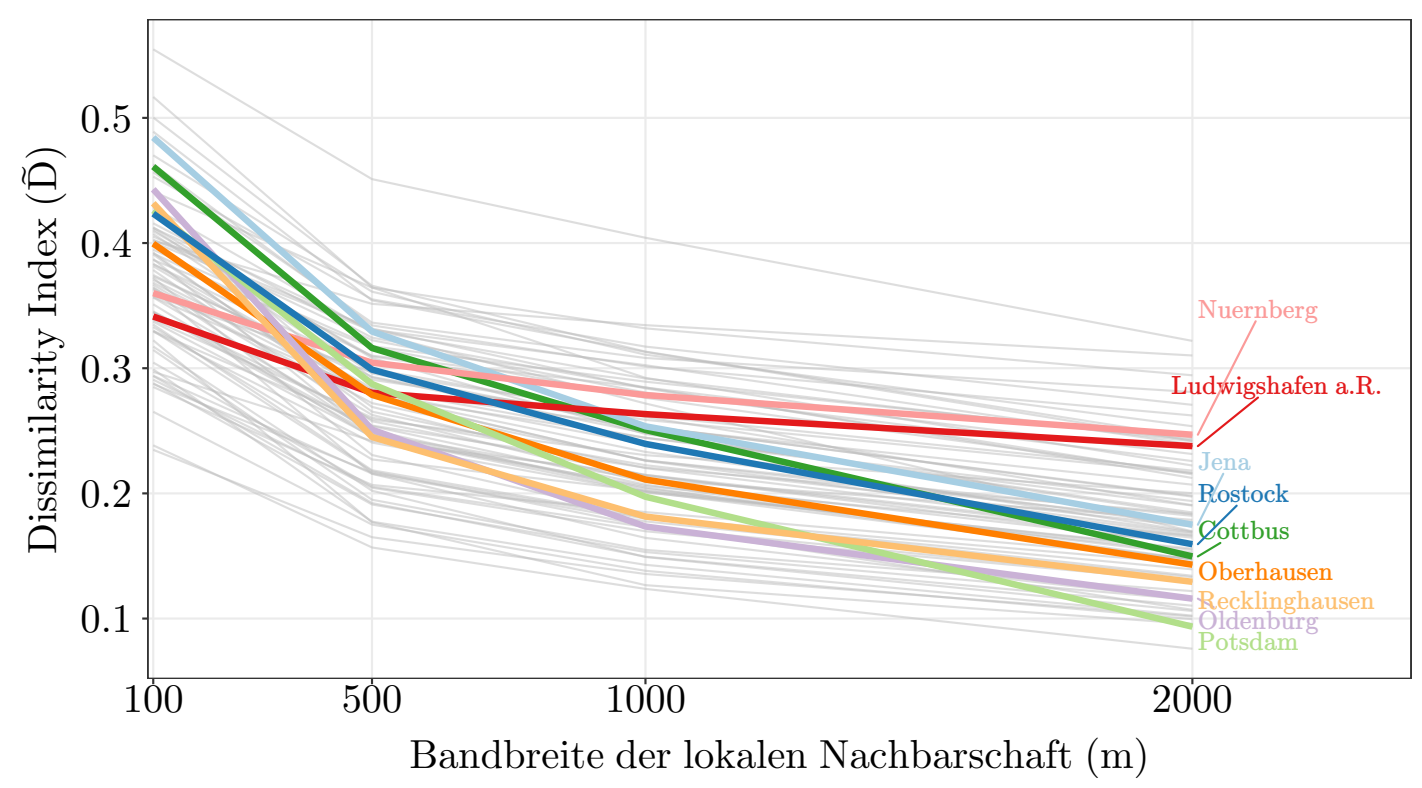

Abbildung 5. Segregations-Profil für deutsche Großstädte.

Neben dem Vergleich zwischen den Städten ist auch der Vergleich der einzelnen Skalen der Segregation innerhalb der Städte interessant. Reardon et al. (2008) verwenden dafür das Segregations-Profil, einen Plot der für jede Stadt auf der Y-Achse das Ausmaß an Segregation und auf der X-Achse die verschiedenen Skalen der lokalen Nachbarschaft abträgt, wie in Abbildung $5 \mathrm{zu}$ sehen. Für alle Städte zeigt der Plot das erwartete Muster: Mit steigender Größe der lokalen Nachbarschaft nimmt das Level an Segregation ab. Für einige Städte lassen sich hier aber auch erstaunliche Muster erkennen. Die Städte Nürnberg und Ludwigshafen am Rhein weisen im Vergleich zu anderen Städten eine relative niedrige Mikro-Segregation auf, gleichzeitig sind die beiden Städte aber relativ stark in großräumige Segregationsmuster unterteilt. Für Recklinghausen und Oldenburg (und weitere markierte Städte) gilt das genaue Gegenteil: Hier lassen sich zwar sehr starke Muster der Mikro-Segregation erkennen, aber eher geringere Werte in der Makro-Segregation.

Um die Unterschiede zwischen den zwei Segregationsmustern zu verdeutlichen, stellt Abbildung 6 die räumliche Verteilung in Ludwigshafen und Oldenburg dar. Ludwigshafen, mit einer geringen Mikro- und starken Makro-Segregation, weist auf der kleinräumigen Ebene kaum Unterschiede im Ausländeranteil auf: Die Übergänge zwischen den Einheiten sehen eher seicht aus. In der Innenstadt scheint ein homogen hoher Ausländeranteil vorzuherrschen. In den Randbezirken im Süden und Westen (Maudach, Rheingönheim und Ruchheim) ist der Ausländeranteil im Gegensatz dazu generell gering, aber auch hier gehen die kleinteiligen Bereiche mit einem höheren Anteil eher seicht in Gebiete mit einem niedrigen Anteil über. Die Trennung scheint hier also eher zwischen den Stadtteilen als zwischen kleinräumigen Gebieten stattzufinden. Oldenburg hingegen weist ein gegenteiliges Muster auf. Obwohl es einige Bereiche mit einem höheren Anteil an Ausländernausländischer Bevölkerung gibt, lassen sich hier kaum ganze Stadtviertel mit einer unterschiedlichen demographischen Zusammensetzung erkennen. Allerdings gibt es in Oldenburg auf der kleinräumigen Ebene (und in allen Stadtteilen) sehr viele „starke Brüche“, also ein kleines Gebiet mit einem Ausländeranteil über 10\% angrenzend an kleinräumige Gebiete mit $0 \%$ Ausländeranteil. Hier scheint eine räumliche 

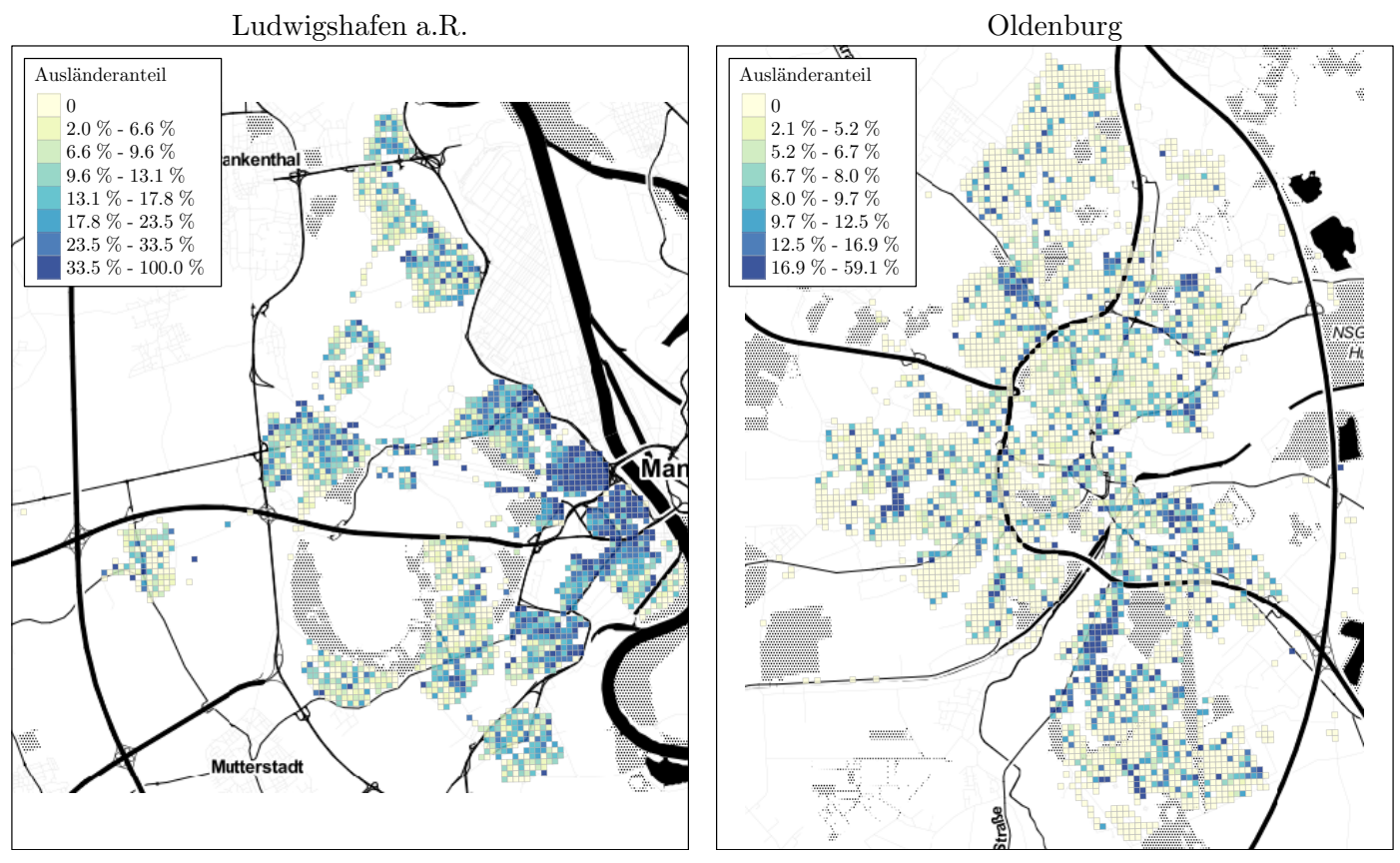

Abbildung 6. Die räumliche Verteilung des Ausländeranteils in Ludwigshafen a.R. und Oldenburg.

Trennung also viel stärker auf einer kleinräumigen geographischen Ebene stattzufinden. Tendenziell scheinen diese Muster - Schwache Makro- und starke Mikro-Segregation — vor allem in Städten mit generell niedrigem Ausländeranteil aufzutreten (Cottbus, Jena, Oldenburg, Potsdam, Rostock). Aber auch Städte mit einem durchschnittlichen Anteil an ausländischen Minderheiten (z.B. Oberhausen und Recklinghausen) zeigen ähnliche Muster.

Diese Beispiele verdeutlichen, wie unterschiedlich die einzelnen Muster der räumlichen Segregation auch in Deutschland ausfallen können. Nicht-räumliche Maße oder Kennzahlen basierend auf einer geographischen Ebene stellen daher eine starke Reduktion der Komplexität von Segregation dar. Besonders für die weitere Untersuchung von Segregation sowie deren Ursachen und Folgen sollten daher auch die räumlichen Muster - sowohl in Theorie als auch Empirie -- Beachtung finden.

\section{Fazit}

Ziel dieses Beitrags war es einen Überblick über das Ausmaß an Segregation und deren Muster in Deutschland zu geben. Dazu verwendet dieser Beitrag Daten des Zensus 2011 und verschiedene räumliche Segregationsmaße. Ein Vorteil der verwendeten Daten liegt vor allem darin, dass sich das Ausmaß an Segregation aufgrund der einheitlichen Messung und räumlichen Gliederung über Stadtgrenzen hinweg vergleichen lässt. Zudem liefern räumliche Segregationsmaße die Möglichkeit, verschiedene Muster von Segregation auf unterschiedlichen räumlichen Skalen zu identifizieren.

Wie bereits in bisherigen Studien dokumentiert (z.B. Helbig \& Jähnen, 2018; Musterd, 2012), weist Deutschland im internationalen Vergleich eher eine geringere räumliche ethnische Segregation auf. Für Personen mit ausländischer Staatsbürgerschaft liegt der Dissimilarity Index im Durchschnitt zwischen 0.38 (100m) und 0.18 
$(2 \mathrm{~km})$ und der Information Theory Index zwischen $0.13(100 \mathrm{~m})$ und $0.03(2 \mathrm{~km})$. Im Vergleich verschiedener Staatsangehörigkeiten zeigt sich, dass auf der kleinräumigen Ebene besonders Einwohner südeuropäischer Staatsangehörigkeit segregiert leben. Auf der Makro-Ebene sind es hingegen Einwohner türkischer Staatsangehörigkeit, die stärker getrennt von Personen deutscher Staatsangehörigkeit wohnen. Folglich sind Personen türkischer Herkunft eher auf einer Ebene von Stadtbezirken oder Gebieten isoliert als andere Gruppen. Im geographischen Vergleich über die Städte hinweg wird deutlich, dass ostdeutsche Städte ein relativ hohes Ausmaß an Segregation aufweisen. Besonders Chemnitz ist auf allen räumlichen Skalen durch ein sehr hohes Niveau an Segregation gekennzeichnet. Aber auch Berlin zeigt ein starkes Ausmaß and großflächiger Segregation. Heidelberg hingegen weist in allen Dimensionen ein extrem niedriges Level an Segregation auf, aber auch in Frankfurt am Main und München ist die räumliche Segregation vergleichsweise gering ausgeprägt.

Eine Erklärung für die starke Heterogenität zwischen den deutschen Großstädten stellt eine interessante Herausforderung für weitere Forschung in diesem Bereich dar. Es liegt auf der Hand, dass die reine Größe der verschiedenen Gruppen an Minderheiten einen starken Einfluss auf die räumlichen Segregationsmuster hat. Besonders für das hohe Level an Segregation in Ostdeutschland mag der vergleichsweise niedrige Anteil an Minderheiten (siehe Tabelle 1) eine große Rolle spielen. Frankfurt am Main und München gehörten 2011 mit 25\% und 21\% zu den Städten mit dem höchsten Ausländeranteil (nach Offenbach am Mai mit 29\%) und zeigen ein hohes Maß an räumlicher Diversität. Ein hoher Anteil allein scheint aber noch nicht vor stärkerer Separierung zu schützen (siehe z.B. Nürnberg und Pforzheim). Vermutlich spielt auch der lokale Arbeitsmarkt und die damit verbundene Sozialstruktur innerhalb der Stadt eine Rolle (Janßen \& Schroedter, 2007). Auch weitere Merkmale, wie z.B. ökonomische Ungleichheiten, der Wohnungsmarkt oder die demographische Zusammensetzung könnten einen Einfluss auf die Stärke der räumlichen Segregation haben (siehe z.B. Helbig \& Jähnen, 2018; Lee et al., 2008). Neben demographischen Merkmalen wäre es aber auch interessant zu untersuchen, inwieweit sich städtische Infrastrukturen und Bauten auf die räumliche Trennung von Bevölkerungsgruppen auswirken (z.B. Roberto, 2018). Unter Verwendung räumlicher Analyseverfahren (Rüttenauer, 2019b) kann außerdem untersucht werden, wie sich der Einfluss von verschiedenen Merkmalen geographisch verändert und so zu einer räumlichen Cluster Bildung führt.

Eine weitere wichtige Erkenntnis aus den vorliegenden Ergebnissen besteht darin, dass Segregation sehr unterschiedliche räumliche Muster annehmen kann. Städte wie z.B. Nürnberg und Ludwigshafen am Rhein weisen eine vergleichsweise niedrige Segregation auf der Mikro-Ebene auf, zeigen aber eher stark ausgeprägte räumliche Unterschiede auf der großräumigen Ebene. Recklinghausen, Oldenburg und Potsdam, zum Beispiel, weisen gegenteiligen Mikro-Makro Muster auf. Das bedeutet, dass auch in Deutschland sehr heterogene Arten der räumlichen Segregation existieren. Gerade für die weitere Analyse von Ursachen und Folgen räumlicher Segregation könnte es von Vorteil sein, zwischen diesen verschiedenen Arten der Segregation zu unterscheiden und so ein besseres Verständnis für die komplexe Interaktion zwischen sozialen und räumlichen Prozessen zu erlangen. 


\section{Literatur}

Alba, R. \& Nee, V. (2005). Remaking the American Mainstream: Assimilation and Contemporary Immigration. Cambridge, Mass.: Harvard Univ. Press.

Auspurg, K., Hinz, T. \& Schmid, L. (2017). Contexts and Conditions of Ethnic Discrimination: Evidence from a Field Experiment in a German Housing Market. Journal of Housing Economics, 35, 26-36.

Auspurg, K., Schneck, A. \& Hinz, T. (2019). Closed Doors Everywhere? A Meta-Analysis of Field Experiments on Ethnic Discrimination in Rental Housing Markets. Journal of Ethnic and Migration Studies, 45 (1), 95-114.

Best, H. \& Rüttenauer, T. (2018). How Selective Migration Shapes Environmental Inequality in Germany: Evidence from Micro-Level Panel Data. European Sociological Review, 34 (1), $52-63$.

Chetty, R., Hendren, N. \& Katz, L. F. (2016). The Effects of Exposure to Better Neighborhoods on Children: New Evidence from the Moving to Opportunity Experiment. American Economic Review, 106 (4), 855-902.

Damm, A. P. (2009). Ethnic Enclaves and Immigrant Labor Market Outcomes: QuasiExperimental Evidence. Journal of Labor Economics, 27 (2), 281-314.

Downey, L. (2007). US Metropolitan-Area Variation in Environmental Inequality Outcomes. Urban Studies, 44 (5-6), 953-977.

Duncan, O. D. \& Duncan, B. (1955). A Methodological Analysis of Segregation Indexes. American Sociological Review, 20 (2), 210.

Firebaugh, G. \& Farrell, C. R. (2016). Still Large, but Narrowing: The Sizable Decline in Racial Neighborhood Inequality in Metropolitan America, 1980-2010. Demography, 53 (1), $139-164$.

Firebaugh, G. \& Schroeder, M. B. (2009). Does Your Neighbor's Income Affect Your Happiness? American Journal of Sociology, 115 (3), 805-831.

Friedrichs, J. (2008). Ethnische Segregation. In F. Kalter (Hrsg.), Migration und Integration (S. 380-411). Wiesbaden: VS Verlag.

Friedrichs, J. \& Triemer, S. (2009). Gespaltene Städte? Soziale und ethnische Segregation in deutschen Großstädten. Wiesbaden: VS Verlag.

Goebel, J. \& Hoppe, L. (2015). Ausmaß und Trends sozialräumlicher Segregation in Deutschland. Berlin. Zugriff auf https://www.armuts-und-reichtumsbericht.de/SharedDocs/ Downloads/Service/Studien/abschlussbericht-sozialraeumliche-segregation.pdf ?_-_blob=publicationFile\&v=3

Helbig, M. \& Jähnen, S. (2018). Wie brüchig ist die soziale Architektur unserer Städte? Trends und Analysen der Segregation in 74 deutschen Städten (Nr. P 2018-001).

Hong, S.-Y., O’Sullivan, D. \& Sadahiro, Y. (2014). Implementing Spatial Segregation Measures in R. PloS one, 9 (11), e113767.

Janßen, A. \& Schroedter, J. H. (2007). Kleinräumliche Segregation der ausländischen Bevölkerung in Deutschland: Eine Analyse auf der Basis des Mikrozensus. Zeitschrift für Soziologie, $36(6), 453-472$.

Klinger, J., Müller, S. \& Schaeffer, M. (2017). Der Halo-Effekt in einheimisch-homogenen Nachbarschaften. Steigert die ethnische Diversität angrenzender Nachbarschaften die Xenophobie in Deutschland? Zeitschrift für Soziologie, 46 (6), 402-419.

Krysan, M. \& Crowder, K. (2017). Cycle of Segregation: Social Processes and Residential Stratification. Chicago: Russell Sage Foundation.

Lee, B. A., Reardon, S. F., Firebaugh, G., Farrell, C. R., Matthews, S. A. \& O'Sullivan, D. (2008). Beyond the Census Tract: Patterns and Determinants of Racial Segregation at Multiple Geographic Scales. American Sociological Review, 73 (5), 766-791.

Legewie, J. \& Schaeffer, M. (2016). Contested Boundaries: Explaining where Ethnoracial Diversity Provokes Neighborhood Conflict. American Journal of Sociology, 122 (1), 125161. 
Lersch, P. M. (2013). Place Stratification or Spatial Assimilation? Neighbourhood Quality Changes after Residential Mobility for Migrants in Germany. Urban Studies, 50 (5), 10111029.

Lichter, D. T., Parisi, D. \& Ambinakudige, S. (2019). The Spatial Integration of Immigrants in Europe: A Cross-National Study. Population Research and Policy Review, 34 (2), 151.

Lichter, D. T., Parisi, D. \& Taquino, M. C. (2015). Toward a New Macro-Segregation? Decomposing Segregation within and between Metropolitan Cities and Suburbs. American Sociological Review, 80 (4), 843-873.

Logan, J. R. (2012). Making a Place for Space: Spatial Thinking in Social Science. Annual Review of Sociology, 38, 507-524.

Logan, J. R. \& Zhang, C. (2010). Global Neighborhoods: New Pathways to Diversity and Separation. American Journal of Sociology, 115 (4), 1069-1109.

Logan, J. R., Zhang, W. \& Alba, R. D. (2002). Immigrant Enclaves and Ethnic Communities in New York and Los Angeles. American Sociological Review, 67 (2), 299-322.

Ludwig, J., Duncan, G. J., Gennetian, L. A., Katz, L. F., Kessler, R. C., Kling, J. R. \& Sanbonmatsu, L. (2012). Neighborhood effects on the long-term well-being of low-income adults. Science, 337 (6101), 1505-1510.

Massey, D. S. \& Denton, N. A. (1988). The Dimensions of Residential Segregation. Social Forces, 67 (2), 281-315.

Massey, D. S. \& Denton, N. A. (1993). American Apartheid: Segregation and the Making of the Underclass. Cambridge, Mass: Harvard Univ. Press.

Musterd, S. (2005). Social and Ethnic Segregation in Europe: Levels, Causes, and Effects. Journal of Urban Affairs, 27 (3), 331-348.

Musterd, S. (2012). Ethnic Segregation - Reflections on Concepts, Levels and Effects. In D. F. Clapham, W. A. V. Clark \& K. Gibb (Hrsg.), The Sage Handbook of Housing Studies (S. 419-438). Los Angeles: Sage.

Phillips, N. E., Levy, B. L., Sampson, R. J., Small, M. L. \& Wang, R. Q. (2019). The Social Integration of American Cities: Network Measures of Connectedness Based on Everyday Mobility Across Neighborhoods. Sociological Methods \& Research, OnlineFirst.

Reardon, S. F. \& Firebaugh, G. (2002). Measures of Multigroup Segregation. Sociological Methodology, 32 (1), 33-67.

Reardon, S. F., Kalogrides, D. \& Shores, K. (2019). The Geography of Racial/Ethnic Test Score Gaps. American Journal of Sociology, 124 (4), 1164-1221.

Reardon, S. F., Matthews, S. A., O’Sullivan, D., Lee, B. A., Firebaugh, G., Farrell, C. R. \& Bischoff, K. (2008). The Geographic Scale of Metropolitan Racial Segregation. Demography, $45(3), 489-514$.

Reardon, S. F. \& O'Sullivan, D. (2004). Measures of Spatial Segregation. Sociological Methodology, 34 (1), 121-162.

Roberto, E. (2018). The Spatial Proximity and Connectivity Method for Measuring and Analyzing Residential Segregation. Sociological Methodology, 48 (1), 182-224.

Rüttenauer, T. (2019a). Bringing Urban Space Back in: A Multilevel Analysis of Environmental Inequality in Germany. Urban Studies, 56 (12), 2549-2567.

Rüttenauer, T. (2019b). Spatial Regression Models: A Systematic Comparison of Different Model Specifications Using Monte Carlo Experiments. Sociological Methods \&6 Research, OnlineFirst.

Sager, L. (2012). Residential Segregation and Socioeconomic Neighbourhood Sorting: Evidence at the Micro-Neighbourhood Level for Migrant Groups in Germany. Urban Studies, 49 (12), 2617-2632.

Sampson, R. J. (2013). Great American City: Chicago and the Enduring Neighborhood Effect. Chicago: University of Chicago Press.

Sharkey, P. \& Faber, J. W. (2014). Where, When, Why, and For Whom Do Residential Contexts Matter? Moving Away from the Dichotomous Understanding of Neighborhood 
Effects. Annual Review of Sociology, 40 (1), 559-579.

Statistische Ämter des Bundes und der Länder. (2015). Zensus 2011: Metoden und Berichte. Wiesbaden. Zugriff am 20.03.2017 auf https://www.zensus2011.de/SharedDocs/ Downloads/DE/Publikationen/Auf saetze_Archiv/2015_06_MethodenUndVerfahren .pdf

Teltemann, J., Dabrowski, S. \& Windzio, M. (2015). Räumliche Segregation von Familien mit Migrationshintergrund in deutschen Großstädten: Wie stark wirkt der sozioökonomische Status? Kölner Zeitschrift für Soziologie und Sozialpsychologie, 67 (1), 83-103.

Tivadar, M. (2019). OasisR : An R Package to Bring Some Order to the World of Segregation Measurement. Journal of Statistical Software, 89 (7), 1-39.

Winke, T. (2018). Being Close to Ethnic Peers: The Formation of Ethnic Residential Clustering from a Household Perspective. Journal of Ethnic and Migration Studies, 44 (11), 19321953.

Wong, D. (2009). The Modifiable Areal Unit Problem (MAUP). In A. S. Fotheringham \& P. Rogerson (Hrsg.), The Sage Handbook of Spatial Analysis (S. 105-124). Los Angeles and London: Sage. 


\section{Anhang A}

Tabelle A1. Segregation für verschiedene Gruppen und lokale Nachbarschaften.

\begin{tabular}{|c|c|c|c|c|c|c|c|c|}
\hline \multirow[b]{2}{*}{ Variable } & \multicolumn{4}{|c|}{ Nationalität: DE - Ausland } & \multicolumn{4}{|c|}{ Geburtsland: DE - Ausland } \\
\hline & Mean & $\mathrm{SD}$ & Min & Max & Mean & $\mathrm{SD}$ & Min & Max \\
\hline$\tilde{D}_{100}$ & 0.377 & 0.063 & 0.235 & 0.555 & 0.301 & 0.039 & 0.217 & 0.441 \\
\hline$\tilde{D}_{500}$ & 0.273 & 0.056 & 0.157 & 0.451 & 0.216 & 0.037 & 0.145 & 0.354 \\
\hline$\tilde{D}_{1000}$ & 0.231 & 0.056 & 0.124 & 0.404 & 0.178 & 0.039 & 0.113 & 0.309 \\
\hline$\tilde{D}_{2000}$ & 0.179 & 0.054 & 0.076 & 0.322 & 0.134 & 0.040 & 0.067 & 0.239 \\
\hline$\tilde{H}_{100}$ & 0.131 & 0.034 & 0.061 & 0.223 & 0.094 & 0.021 & 0.052 & 0.172 \\
\hline$\tilde{H}_{500}$ & 0.065 & 0.023 & 0.026 & 0.133 & 0.046 & 0.013 & 0.023 & 0.093 \\
\hline$\tilde{H}_{1000}$ & 0.046 & 0.019 & 0.015 & 0.106 & 0.032 & 0.012 & 0.013 & 0.066 \\
\hline$\tilde{H}_{2000}$ & 0.029 & 0.016 & 0.005 & 0.081 & 0.019 & 0.010 & 0.005 & 0.049 \\
\hline Macro-Micro Ratio $\tilde{D}$ & 0.472 & 0.106 & 0.219 & 0.772 & 0.443 & 0.103 & 0.213 & 0.723 \\
\hline \multirow[t]{2}{*}{ Macro-Micro Ratio $\tilde{H}$} & 0.217 & 0.093 & 0.059 & 0.552 & 0.199 & 0.084 & 0.051 & 0.499 \\
\hline & \multicolumn{4}{|c|}{ Nationalität: DE - Südeuropa } & \multicolumn{4}{|c|}{ Nationalität: DE - Osteuropa } \\
\hline Variable & Mean & $\mathrm{SD}$ & Min & Max & Mean & SD & Min & Max \\
\hline$\tilde{D}_{100}$ & 0.651 & 0.184 & 0.330 & 0.980 & 0.560 & 0.099 & 0.336 & 0.770 \\
\hline$\tilde{D}_{500}$ & 0.387 & 0.137 & 0.182 & 0.814 & 0.333 & 0.062 & 0.211 & 0.541 \\
\hline$\tilde{D}_{1000}$ & 0.308 & 0.102 & 0.152 & 0.627 & 0.270 & 0.059 & 0.153 & 0.471 \\
\hline$\tilde{D}_{2000}$ & 0.235 & 0.079 & 0.106 & 0.424 & 0.203 & 0.059 & 0.083 & 0.411 \\
\hline$\tilde{H}_{100}$ & 0.231 & 0.096 & 0.088 & 0.498 & 0.190 & 0.043 & 0.100 & 0.290 \\
\hline$\tilde{H}_{500}$ & 0.083 & 0.045 & 0.027 & 0.270 & 0.069 & 0.022 & 0.038 & 0.150 \\
\hline$\tilde{H}_{1000}$ & 0.052 & 0.027 & 0.015 & 0.145 & 0.045 & 0.018 & 0.016 & 0.111 \\
\hline$\tilde{H}_{2000}$ & 0.030 & 0.017 & 0.006 & 0.085 & 0.026 & 0.013 & 0.005 & 0.071 \\
\hline Macro-Micro Ratio $\tilde{D}$ & 0.371 & 0.106 & 0.154 & 0.663 & 0.368 & 0.099 & 0.136 & 0.592 \\
\hline \multirow[t]{2}{*}{ Macro-Micro Ratio $\tilde{H}$} & 0.137 & 0.075 & 0.029 & 0.420 & 0.139 & 0.065 & 0.029 & 0.332 \\
\hline & \multicolumn{4}{|c|}{ Nationalität: DE - Türkei } & \multicolumn{4}{|c|}{ Nationalität: Multigroup } \\
\hline Variable & Mean & $\mathrm{SD}$ & Min & $\operatorname{Max}$ & Mean & $\mathrm{SD}$ & Min & Max \\
\hline$\tilde{D}_{100}$ & 0.595 & 0.136 & 0.381 & 0.941 & 0.446 & 0.073 & 0.270 & 0.621 \\
\hline$\tilde{D}_{500}$ & 0.414 & 0.093 & 0.253 & 0.671 & 0.309 & 0.061 & 0.176 & 0.489 \\
\hline$\tilde{D}_{1000}$ & 0.345 & 0.084 & 0.180 & 0.570 & 0.260 & 0.059 & 0.144 & 0.434 \\
\hline$\tilde{D}_{2000}$ & 0.266 & 0.078 & 0.117 & 0.486 & 0.201 & 0.055 & 0.101 & 0.344 \\
\hline$\tilde{H}_{100}$ & 0.227 & 0.060 & 0.118 & 0.415 & 0.175 & 0.042 & 0.084 & 0.264 \\
\hline$\tilde{H}_{500}$ & 0.109 & 0.036 & 0.046 & 0.235 & 0.076 & 0.024 & 0.032 & 0.147 \\
\hline$\tilde{H}_{1000}$ & 0.076 & 0.031 & 0.025 & 0.179 & 0.052 & 0.019 & 0.021 & 0.107 \\
\hline$\tilde{H}_{2000}$ & 0.046 & 0.024 & 0.011 & 0.144 & 0.032 & 0.015 & 0.011 & 0.080 \\
\hline Macro-Micro Ratio $\tilde{D}$ & 0.453 & 0.111 & 0.161 & 0.755 & 0.449 & 0.094 & 0.235 & 0.735 \\
\hline Macro-Micro Ratio $\tilde{H}$ & 0.206 & 0.093 & 0.032 & 0.550 & 0.181 & 0.071 & 0.067 & 0.471 \\
\hline
\end{tabular}


Tabelle A2.: Segregation: Dissimilarity Index $\tilde{D}$

\begin{tabular}{|c|c|c|c|c|c|c|c|c|c|}
\hline \multirow[b]{2}{*}{ Stadt } & \multicolumn{4}{|c|}{$\tilde{D}_{100}$} & \multicolumn{4}{|c|}{$\tilde{D}_{2000}$} & \multirow{2}{*}{$\begin{array}{l}\text { Ratio } \\
\mathrm{D}-\mathrm{A}\end{array}$} \\
\hline & $\mathrm{D}-\mathrm{A}$ & $D-S$ & $\mathrm{D}-\mathrm{O}$ & $\mathrm{D}-\mathrm{T}$ & $D-A$ & $\mathrm{D}-\mathrm{S}$ & $\mathrm{D}-\mathrm{O}$ & $\mathrm{D}-\mathrm{T}$ & \\
\hline Chemnitz & 0.555 & 0.960 & 0.708 & 0.941 & 0.322 & 0.319 & 0.366 & 0.333 & 0.580 \\
\hline Erfurt & 0.517 & 0.956 & 0.717 & 0.851 & 0.222 & 0.419 & 0.235 & 0.302 & 0.430 \\
\hline Magdeburg & 0.500 & 0.941 & 0.675 & 0.896 & 0.245 & 0.351 & 0.249 & 0.368 & 0.489 \\
\hline Halle (Saale) & 0.489 & 0.944 & 0.698 & 0.835 & 0.242 & 0.398 & 0.313 & 0.326 & 0.495 \\
\hline Jena & 0.484 & 0.886 & 0.724 & 0.913 & 0.175 & 0.297 & 0.411 & 0.147 & 0.361 \\
\hline Hamm & 0.470 & 0.887 & 0.597 & 0.582 & 0.254 & 0.416 & 0.237 & 0.309 & 0.540 \\
\hline Bottrop & 0.463 & 0.774 & 0.756 & 0.588 & 0.262 & 0.208 & 0.194 & 0.357 & 0.567 \\
\hline Cottbus & 0.461 & 0.974 & 0.635 & 0.915 & 0.150 & 0.403 & 0.217 & 0.250 & 0.325 \\
\hline Dresden & 0.457 & 0.906 & 0.659 & 0.887 & 0.281 & 0.295 & 0.297 & 0.431 & 0.615 \\
\hline Leipzig & 0.453 & 0.882 & 0.628 & 0.891 & 0.271 & 0.262 & 0.301 & 0.486 & 0.598 \\
\hline Oldenburg & 0.443 & 0.940 & 0.770 & 0.706 & 0.116 & 0.231 & 0.172 & 0.212 & 0.262 \\
\hline Dortmund & 0.441 & 0.679 & 0.553 & 0.594 & 0.294 & 0.417 & 0.285 & 0.392 & 0.667 \\
\hline Recklinghausen & 0.432 & 0.826 & 0.617 & 0.551 & 0.129 & 0.127 & 0.169 & 0.210 & 0.300 \\
\hline Moers & 0.429 & 0.698 & 0.681 & 0.614 & 0.170 & 0.209 & 0.142 & 0.287 & 0.396 \\
\hline Potsdam & 0.426 & 0.964 & 0.609 & 0.864 & 0.094 & 0.393 & 0.146 & 0.268 & 0.219 \\
\hline Rostock & 0.423 & 0.980 & 0.570 & 0.858 & 0.159 & 0.284 & 0.244 & 0.373 & 0.376 \\
\hline Siegen & 0.423 & 0.792 & 0.723 & 0.628 & 0.182 & 0.203 & 0.226 & 0.315 & 0.430 \\
\hline Kiel & 0.416 & 0.909 & 0.594 & 0.603 & 0.212 & 0.333 & 0.243 & 0.350 & 0.511 \\
\hline Hagen & 0.413 & 0.520 & 0.558 & 0.534 & 0.185 & 0.211 & 0.188 & 0.224 & 0.448 \\
\hline Hildesheim & 0.412 & 0.741 & 0.649 & 0.570 & 0.239 & 0.203 & 0.213 & 0.335 & 0.580 \\
\hline Muelheim a.d.R. & 0.411 & 0.699 & 0.572 & 0.570 & 0.198 & 0.188 & 0.183 & 0.281 & 0.482 \\
\hline Essen & 0.409 & 0.670 & 0.546 & 0.566 & 0.246 & 0.243 & 0.238 & 0.361 & 0.601 \\
\hline Bochum & 0.407 & 0.722 & 0.605 & 0.552 & 0.183 & 0.201 & 0.175 & 0.238 & 0.450 \\
\hline Duisburg & 0.405 & 0.640 & 0.565 & 0.508 & 0.226 & 0.300 & 0.247 & 0.282 & 0.557 \\
\hline Pforzheim & 0.403 & 0.472 & 0.440 & 0.506 & 0.215 & 0.225 & 0.196 & 0.235 & 0.534 \\
\hline Berlin & 0.402 & 0.639 & 0.470 & 0.626 & 0.310 & 0.424 & 0.278 & 0.472 & 0.772 \\
\hline Krefeld & 0.401 & 0.555 & 0.573 & 0.546 & 0.242 & 0.288 & 0.287 & 0.288 & 0.603 \\
\hline Oberhausen & 0.400 & 0.581 & 0.575 & 0.524 & 0.143 & 0.214 & 0.176 & 0.200 & 0.358 \\
\hline Osnabrueck & 0.399 & 0.831 & 0.600 & 0.656 & 0.169 & 0.171 & 0.178 & 0.361 & 0.423 \\
\hline Ingolstadt & 0.395 & 0.715 & 0.572 & 0.619 & 0.243 & 0.285 & 0.280 & 0.348 & 0.616 \\
\hline Bielefeld & 0.392 & 0.667 & 0.608 & 0.532 & 0.168 & 0.320 & 0.187 & 0.224 & 0.428 \\
\hline Koblenz & 0.392 & 0.785 & 0.552 & 0.670 & 0.231 & 0.248 & 0.214 & 0.453 & 0.591 \\
\hline Luebeck & 0.391 & 0.813 & 0.640 & 0.567 & 0.165 & 0.242 & 0.239 & 0.275 & 0.421 \\
\hline Bergisch Gladbach & 0.387 & 0.669 & 0.695 & 0.606 & 0.151 & 0.180 & 0.161 & 0.252 & 0.390 \\
\hline Kassel & 0.386 & 0.700 & 0.559 & 0.547 & 0.216 & 0.225 & 0.206 & 0.312 & 0.560 \\
\hline Wuppertal & 0.383 & 0.475 & 0.502 & 0.518 & 0.190 & 0.207 & 0.191 & 0.229 & 0.496 \\
\hline Bremerhaven & 0.383 & 0.904 & 0.586 & 0.549 & 0.191 & 0.314 & 0.129 & 0.296 & 0.498 \\
\hline Remscheid & 0.381 & 0.478 & 0.613 & 0.536 & 0.174 & 0.126 & 0.156 & 0.288 & 0.457 \\
\hline Paderborn & 0.378 & 0.722 & 0.685 & 0.655 & 0.160 & 0.289 & 0.208 & 0.289 & 0.422 \\
\hline Braunschweig & 0.374 & 0.715 & 0.578 & 0.545 & 0.216 & 0.242 & 0.242 & 0.261 & 0.578 \\
\hline Fuerth & 0.373 & 0.508 & 0.521 & 0.471 & 0.197 & 0.234 & 0.232 & 0.215 & 0.529 \\
\hline Muenster & 0.372 & 0.808 & 0.642 & 0.717 & 0.134 & 0.163 & 0.203 & 0.247 & 0.360 \\
\hline Moenchengladbach & 0.371 & 0.679 & 0.611 & 0.523 & 0.207 & 0.219 & 0.238 & 0.280 & 0.559 \\
\hline Goettingen & 0.368 & 0.717 & 0.600 & 0.631 & 0.194 & 0.141 & 0.212 & 0.301 & 0.526 \\
\hline Gelsenkirchen & 0.367 & 0.685 & 0.536 & 0.448 & 0.160 & 0.290 & 0.246 & 0.178 & 0.436 \\
\hline Solingen & 0.365 & 0.470 & 0.665 & 0.513 & 0.145 & 0.192 & 0.194 & 0.185 & 0.398 \\
\hline Neuss & 0.364 & 0.574 & 0.516 & 0.529 & 0.177 & 0.316 & 0.192 & 0.194 & 0.486 \\
\hline Herne & 0.363 & 0.608 & 0.582 & 0.455 & 0.115 & 0.106 & 0.136 & 0.153 & 0.317 \\
\hline Nuernberg & 0.360 & 0.491 & 0.436 & 0.481 & 0.247 & 0.321 & 0.242 & 0.294 & 0.686 \\
\hline Mannheim & 0.358 & 0.448 & 0.420 & 0.514 & 0.218 & 0.234 & 0.211 & 0.272 & 0.609 \\
\hline Heilbronn & 0.357 & 0.478 & 0.439 & 0.444 & 0.169 & 0.172 & 0.177 & 0.188 & 0.475 \\
\hline Bremen & 0.357 & 0.770 & 0.532 & 0.526 & 0.147 & 0.246 & 0.180 & 0.273 & 0.413 \\
\hline Wolfsburg & 0.351 & 0.448 & 0.695 & 0.780 & 0.166 & 0.184 & 0.230 & 0.310 & 0.473 \\
\hline Saarbruecken & 0.345 & 0.517 & 0.576 & 0.656 & 0.155 & 0.161 & 0.262 & 0.248 & 0.448 \\
\hline Hamburg & 0.345 & 0.658 & 0.493 & 0.552 & 0.200 & 0.294 & 0.248 & 0.356 & 0.581 \\
\hline Reutlingen & 0.345 & 0.475 & 0.511 & 0.529 & 0.179 & 0.246 & 0.187 & 0.186 & 0.519 \\
\hline Ludwigshafen a.R. & 0.341 & 0.387 & 0.457 & 0.439 & 0.238 & 0.208 & 0.236 & 0.241 & 0.697 \\
\hline Aachen & 0.339 & 0.616 & 0.498 & 0.539 & 0.174 & 0.249 & 0.201 & 0.310 & 0.513 \\
\hline Hannover & 0.337 & 0.539 & 0.453 & 0.496 & 0.147 & 0.228 & 0.142 & 0.270 & 0.436 \\
\hline Ulm & 0.335 & 0.456 & 0.469 & 0.480 & 0.144 & 0.168 & 0.158 & 0.182 & 0.430 \\
\hline Erlangen & 0.333 & 0.616 & 0.574 & 0.656 & 0.153 & 0.176 & 0.196 & 0.312 & 0.459 \\
\hline Wuerzburg & 0.330 & 0.654 & 0.578 & 0.651 & 0.107 & 0.157 & 0.217 & 0.233 & 0.324 \\
\hline Koeln & 0.330 & 0.417 & 0.504 & 0.489 & 0.184 & 0.190 & 0.261 & 0.297 & 0.557 \\
\hline
\end{tabular}


Tabelle A2.: Segregation: Dissimilarity Index $\tilde{D}$

\begin{tabular}{|c|c|c|c|c|c|c|c|c|c|}
\hline \multirow[b]{2}{*}{ Stadt } & \multicolumn{4}{|c|}{$\tilde{D}_{100}$} & \multicolumn{4}{|c|}{$\tilde{D}_{2000}$} & \multirow{2}{*}{$\begin{array}{l}\text { Ratio } \\
\mathrm{D}-\mathrm{A}\end{array}$} \\
\hline & $\mathrm{D}-\mathrm{A}$ & $D-S$ & $\mathrm{D}-\mathrm{O}$ & $\mathrm{D}-\mathrm{T}$ & $\mathrm{D}-\mathrm{A}$ & $D-S$ & $\mathrm{D}-\mathrm{O}$ & $\mathrm{D}-\mathrm{T}$ & \\
\hline Leverkusen & 0.329 & 0.473 & 0.544 & 0.504 & 0.156 & 0.184 & 0.144 & 0.187 & 0.473 \\
\hline Regensburg & 0.322 & 0.814 & 0.524 & 0.592 & 0.096 & 0.207 & 0.118 & 0.156 & 0.297 \\
\hline Mainz & 0.317 & 0.420 & 0.463 & 0.524 & 0.106 & 0.137 & 0.090 & 0.176 & 0.334 \\
\hline Bonn & 0.315 & 0.641 & 0.568 & 0.602 & 0.122 & 0.137 & 0.124 & 0.256 & 0.388 \\
\hline Augsburg & 0.305 & 0.452 & 0.435 & 0.424 & 0.130 & 0.185 & 0.124 & 0.144 & 0.428 \\
\hline Trier & 0.299 & 0.843 & 0.610 & 0.805 & 0.102 & 0.244 & 0.083 & 0.198 & 0.342 \\
\hline Offenbach a.M. & 0.297 & 0.351 & 0.351 & 0.381 & 0.166 & 0.177 & 0.183 & 0.160 & 0.560 \\
\hline Duesseldorf & 0.294 & 0.429 & 0.424 & 0.527 & 0.133 & 0.180 & 0.174 & 0.246 & 0.453 \\
\hline Darmstadt & 0.294 & 0.440 & 0.464 & 0.454 & 0.110 & 0.143 & 0.115 & 0.117 & 0.375 \\
\hline Stuttgart & 0.291 & 0.388 & 0.366 & 0.470 & 0.130 & 0.182 & 0.145 & 0.221 & 0.446 \\
\hline Freiburg i.B. & 0.288 & 0.463 & 0.495 & 0.595 & 0.115 & 0.139 & 0.202 & 0.198 & 0.399 \\
\hline Wiesbaden & 0.287 & 0.464 & 0.444 & 0.464 & 0.139 & 0.235 & 0.195 & 0.181 & 0.485 \\
\hline Karlsruhe & 0.285 & 0.467 & 0.411 & 0.476 & 0.148 & 0.173 & 0.158 & 0.198 & 0.519 \\
\hline Muenchen & 0.265 & 0.367 & 0.364 & 0.523 & 0.100 & 0.149 & 0.125 & 0.230 & 0.375 \\
\hline Heidelberg & 0.238 & 0.455 & 0.461 & 0.491 & 0.076 & 0.145 & 0.145 & 0.212 & 0.318 \\
\hline Frankfurt a.M. & 0.235 & 0.330 & 0.336 & 0.420 & 0.102 & 0.120 & 0.128 & 0.189 & 0.433 \\
\hline
\end{tabular}

Tabelle A3.: Segregation: Information Theory Index $\tilde{H}$

\begin{tabular}{|c|c|c|c|c|c|c|c|c|c|}
\hline \multirow[b]{2}{*}{ Stadt } & \multicolumn{4}{|c|}{$\tilde{H}_{100}$} & \multicolumn{4}{|c|}{$\tilde{H}_{2000}$} & \multirow{2}{*}{$\begin{array}{l}\text { Ratio } \\
\mathrm{D}-\mathrm{A}\end{array}$} \\
\hline & $\mathrm{D}-\mathrm{A}$ & $\mathrm{D}-\mathrm{S}$ & $\mathrm{D}-\mathrm{O}$ & $\mathrm{D}-\mathrm{T}$ & $\mathrm{D}-\mathrm{A}$ & $\mathrm{D}-\mathrm{S}$ & $\mathrm{D}-\mathrm{O}$ & $\mathrm{D}-\mathrm{T}$ & \\
\hline Chemnitz & 0.223 & 0.398 & 0.274 & 0.415 & 0.064 & 0.041 & 0.071 & 0.042 & 0.288 \\
\hline Magdeburg & 0.206 & 0.386 & 0.249 & 0.358 & 0.040 & 0.046 & 0.031 & 0.052 & 0.192 \\
\hline Erfurt & 0.205 & 0.436 & 0.288 & 0.296 & 0.035 & 0.059 & 0.034 & 0.040 & 0.169 \\
\hline Jena & 0.205 & 0.339 & 0.263 & 0.341 & 0.023 & 0.032 & 0.070 & 0.011 & 0.113 \\
\hline Hamm & 0.199 & 0.361 & 0.224 & 0.270 & 0.056 & 0.064 & 0.031 & 0.079 & 0.282 \\
\hline Halle (Saale) & 0.187 & 0.402 & 0.239 & 0.319 & 0.036 & 0.054 & 0.045 & 0.040 & 0.190 \\
\hline Dortmund & 0.180 & 0.241 & 0.205 & 0.259 & 0.081 & 0.085 & 0.050 & 0.105 & 0.451 \\
\hline Bottrop & 0.179 & 0.276 & 0.256 & 0.257 & 0.054 & 0.020 & 0.018 & 0.093 & 0.300 \\
\hline Rostock & 0.169 & 0.498 & 0.200 & 0.295 & 0.019 & 0.030 & 0.030 & 0.048 & 0.114 \\
\hline Leipzig & 0.168 & 0.326 & 0.217 & 0.396 & 0.052 & 0.029 & 0.050 & 0.096 & 0.307 \\
\hline Dresden & 0.167 & 0.344 & 0.218 & 0.330 & 0.050 & 0.034 & 0.044 & 0.064 & 0.297 \\
\hline Cottbus & 0.163 & 0.435 & 0.203 & 0.365 & 0.018 & 0.056 & 0.024 & 0.032 & 0.112 \\
\hline Moers & 0.163 & 0.227 & 0.230 & 0.297 & 0.026 & 0.019 & 0.012 & 0.061 & 0.161 \\
\hline Siegen & 0.161 & 0.294 & 0.258 & 0.257 & 0.028 & 0.021 & 0.024 & 0.063 & 0.173 \\
\hline Oldenburg & 0.160 & 0.402 & 0.290 & 0.259 & 0.012 & 0.021 & 0.016 & 0.022 & 0.074 \\
\hline Duisburg & 0.159 & 0.237 & 0.208 & 0.219 & 0.049 & 0.052 & 0.035 & 0.067 & 0.305 \\
\hline Recklinghausen & 0.158 & 0.288 & 0.214 & 0.235 & 0.016 & 0.008 & 0.018 & 0.034 & 0.100 \\
\hline Hagen & 0.151 & 0.184 & 0.173 & 0.212 & 0.030 & 0.029 & 0.021 & 0.038 & 0.200 \\
\hline Muelheim a.d.R. & 0.151 & 0.232 & 0.188 & 0.231 & 0.031 & 0.016 & 0.018 & 0.051 & 0.207 \\
\hline Potsdam & 0.150 & 0.495 & 0.203 & 0.306 & 0.009 & 0.071 & 0.013 & 0.035 & 0.059 \\
\hline Oberhausen & 0.147 & 0.181 & 0.208 & 0.221 & 0.018 & 0.023 & 0.019 & 0.030 & 0.121 \\
\hline Berlin & 0.147 & 0.188 & 0.146 & 0.263 & 0.081 & 0.079 & 0.048 & 0.144 & 0.552 \\
\hline Hildesheim & 0.145 & 0.242 & 0.226 & 0.208 & 0.038 & 0.018 & 0.023 & 0.062 & 0.265 \\
\hline Bochum & 0.145 & 0.238 & 0.210 & 0.208 & 0.028 & 0.019 & 0.022 & 0.037 & 0.192 \\
\hline Essen & 0.144 & 0.219 & 0.178 & 0.224 & 0.046 & 0.028 & 0.035 & 0.079 & 0.319 \\
\hline Kiel & 0.144 & 0.353 & 0.194 & 0.228 & 0.032 & 0.042 & 0.029 & 0.068 & 0.225 \\
\hline Bielefeld & 0.143 & 0.243 & 0.203 & 0.213 & 0.024 & 0.052 & 0.021 & 0.041 & 0.169 \\
\hline Pforzheim & 0.142 & 0.155 & 0.146 & 0.182 & 0.042 & 0.035 & 0.024 & 0.043 & 0.297 \\
\hline Krefeld & 0.139 & 0.186 & 0.194 & 0.221 & 0.044 & 0.041 & 0.040 & 0.060 & 0.315 \\
\hline Kassel & 0.139 & 0.237 & 0.186 & 0.230 & 0.039 & 0.022 & 0.025 & 0.062 & 0.284 \\
\hline Osnabrueck & 0.138 & 0.305 & 0.218 & 0.260 & 0.023 & 0.015 & 0.016 & 0.068 & 0.166 \\
\hline Paderborn & 0.137 & 0.260 & 0.290 & 0.281 & 0.025 & 0.041 & 0.037 & 0.052 & 0.181 \\
\hline Remscheid & 0.135 & 0.153 & 0.197 & 0.225 & 0.024 & 0.012 & 0.014 & 0.054 & 0.179 \\
\hline Bremerhaven & 0.135 & 0.385 & 0.177 & 0.222 & 0.029 & 0.041 & 0.011 & 0.066 & 0.213 \\
\hline Ingolstadt & 0.135 & 0.267 & 0.197 & 0.259 & 0.048 & 0.036 & 0.048 & 0.079 & 0.355 \\
\hline Wuppertal & 0.133 & 0.162 & 0.161 & 0.193 & 0.032 & 0.028 & 0.023 & 0.045 & 0.242 \\
\hline Bergisch Gladbach & 0.133 & 0.240 & 0.236 & 0.233 & 0.018 & 0.017 & 0.013 & 0.039 & 0.134 \\
\hline Koblenz & 0.132 & 0.266 & 0.183 & 0.268 & 0.038 & 0.027 & 0.025 & 0.104 & 0.290 \\
\hline Goettingen & 0.130 & 0.232 & 0.200 & 0.217 & 0.027 & 0.009 & 0.021 & 0.047 & 0.208 \\
\hline
\end{tabular}


Tabelle A3.: Segregation: Information Theory Index $\tilde{H}$

\begin{tabular}{|c|c|c|c|c|c|c|c|c|c|}
\hline \multirow[b]{2}{*}{ Stadt } & \multicolumn{4}{|c|}{$\tilde{H}_{100}$} & \multicolumn{4}{|c|}{$\tilde{H}_{2000}$} & \multirow{2}{*}{$\begin{array}{l}\text { Ratio } \\
\mathrm{D}-\mathrm{A}\end{array}$} \\
\hline & $\mathrm{D}-\mathrm{A}$ & $\mathrm{D}-\mathrm{S}$ & $\mathrm{D}-\mathrm{O}$ & $\mathrm{D}-\mathrm{T}$ & $\mathrm{D}-\mathrm{A}$ & $\mathrm{D}-\mathrm{S}$ & $\mathrm{D}-\mathrm{O}$ & $\mathrm{D}-\mathrm{T}$ & \\
\hline Muenster & 0.128 & 0.268 & 0.215 & 0.253 & 0.016 & 0.013 & 0.022 & 0.032 & 0.125 \\
\hline Braunschweig & 0.127 & 0.234 & 0.181 & 0.195 & 0.033 & 0.026 & 0.035 & 0.041 & 0.259 \\
\hline Luebeck & 0.126 & 0.310 & 0.214 & 0.201 & 0.021 & 0.045 & 0.034 & 0.043 & 0.166 \\
\hline Gelsenkirchen & 0.125 & 0.228 & 0.176 & 0.172 & 0.022 & 0.035 & 0.029 & 0.026 & 0.172 \\
\hline Fuerth & 0.124 & 0.179 & 0.198 & 0.160 & 0.032 & 0.032 & 0.029 & 0.034 & 0.255 \\
\hline Nuernberg & 0.124 & 0.179 & 0.141 & 0.170 & 0.054 & 0.061 & 0.043 & 0.060 & 0.438 \\
\hline Mannheim & 0.123 & 0.144 & 0.134 & 0.205 & 0.046 & 0.035 & 0.030 & 0.057 & 0.373 \\
\hline Bremen & 0.123 & 0.258 & 0.175 & 0.206 & 0.023 & 0.025 & 0.018 & 0.059 & 0.183 \\
\hline Solingen & 0.123 & 0.162 & 0.222 & 0.194 & 0.021 & 0.028 & 0.017 & 0.029 & 0.169 \\
\hline Heilbronn & 0.122 & 0.155 & 0.140 & 0.168 & 0.027 & 0.019 & 0.021 & 0.029 & 0.219 \\
\hline Herne & 0.121 & 0.193 & 0.227 & 0.171 & 0.012 & 0.006 & 0.012 & 0.019 & 0.100 \\
\hline Neuss & 0.121 & 0.197 & 0.155 & 0.209 & 0.030 & 0.053 & 0.021 & 0.026 & 0.248 \\
\hline Moenchengladbach & 0.121 & 0.228 & 0.217 & 0.183 & 0.032 & 0.025 & 0.031 & 0.048 & 0.268 \\
\hline Reutlingen & 0.116 & 0.174 & 0.169 & 0.191 & 0.027 & 0.040 & 0.023 & 0.026 & 0.236 \\
\hline Hamburg & 0.116 & 0.204 & 0.162 & 0.211 & 0.042 & 0.041 & 0.039 & 0.092 & 0.361 \\
\hline Saarbruecken & 0.114 & 0.173 & 0.206 & 0.245 & 0.021 & 0.015 & 0.042 & 0.034 & 0.182 \\
\hline Wolfsburg & 0.111 & 0.152 & 0.224 & 0.281 & 0.025 & 0.026 & 0.028 & 0.042 & 0.221 \\
\hline Ludwigshafen a.R. & 0.111 & 0.117 & 0.140 & 0.155 & 0.045 & 0.032 & 0.030 & 0.040 & 0.406 \\
\hline Ulm & 0.110 & 0.135 & 0.158 & 0.175 & 0.026 & 0.021 & 0.023 & 0.030 & 0.234 \\
\hline Aachen & 0.110 & 0.192 & 0.153 & 0.207 & 0.028 & 0.029 & 0.026 & 0.058 & 0.253 \\
\hline Hannover & 0.107 & 0.164 & 0.147 & 0.177 & 0.018 & 0.025 & 0.013 & 0.043 & 0.169 \\
\hline Koeln & 0.107 & 0.121 & 0.181 & 0.185 & 0.032 & 0.023 & 0.042 & 0.064 & 0.299 \\
\hline Erlangen & 0.105 & 0.199 & 0.222 & 0.253 & 0.020 & 0.017 & 0.023 & 0.051 & 0.187 \\
\hline Leverkusen & 0.102 & 0.144 & 0.176 & 0.163 & 0.019 & 0.018 & 0.013 & 0.020 & 0.186 \\
\hline Regensburg & 0.101 & 0.279 & 0.170 & 0.211 & 0.010 & 0.021 & 0.010 & 0.016 & 0.095 \\
\hline Wuerzburg & 0.097 & 0.202 & 0.200 & 0.234 & 0.010 & 0.012 & 0.031 & 0.027 & 0.098 \\
\hline Bonn & 0.095 & 0.205 & 0.189 & 0.230 & 0.014 & 0.010 & 0.009 & 0.039 & 0.143 \\
\hline Mainz & 0.095 & 0.123 & 0.151 & 0.196 & 0.011 & 0.012 & 0.005 & 0.022 & 0.112 \\
\hline Augsburg & 0.094 & 0.133 & 0.140 & 0.144 & 0.016 & 0.020 & 0.013 & 0.015 & 0.172 \\
\hline Offenbach a.M. & 0.090 & 0.104 & 0.100 & 0.118 & 0.027 & 0.022 & 0.024 & 0.019 & 0.304 \\
\hline Stuttgart & 0.087 & 0.122 & 0.108 & 0.164 & 0.016 & 0.023 & 0.015 & 0.031 & 0.183 \\
\hline Darmstadt & 0.086 & 0.125 & 0.147 & 0.147 & 0.011 & 0.010 & 0.009 & 0.011 & 0.125 \\
\hline Duesseldorf & 0.085 & 0.131 & 0.127 & 0.191 & 0.018 & 0.023 & 0.022 & 0.039 & 0.211 \\
\hline Trier & 0.082 & 0.295 & 0.195 & 0.279 & 0.008 & 0.024 & 0.006 & 0.025 & 0.096 \\
\hline Wiesbaden & 0.082 & 0.152 & 0.139 & 0.160 & 0.020 & 0.039 & 0.025 & 0.029 & 0.251 \\
\hline Karlsruhe & 0.081 & 0.137 & 0.126 & 0.156 & 0.020 & 0.016 & 0.019 & 0.028 & 0.252 \\
\hline Freiburg i.B. & 0.080 & 0.135 & 0.161 & 0.197 & 0.012 & 0.011 & 0.023 & 0.021 & 0.144 \\
\hline Muenchen & 0.072 & 0.106 & 0.107 & 0.190 & 0.011 & 0.015 & 0.012 & 0.039 & 0.150 \\
\hline Heidelberg & 0.064 & 0.128 & 0.149 & 0.161 & 0.005 & 0.012 & 0.016 & 0.024 & 0.085 \\
\hline Frankfurt a.M. & 0.061 & 0.088 & 0.104 & 0.134 & 0.011 & 0.010 & 0.014 & 0.025 & 0.184 \\
\hline
\end{tabular}

